\title{
Review Article \\ Evaluating pH in the Extracellular Tumor Microenvironment Using CEST MRI and Other Imaging Methods
}

\author{
Liu Qi Chen ${ }^{1}$ and Mark D. Pagel ${ }^{1,2,3,4}$ \\ ${ }^{1}$ Department of Chemistry and Biochemistry, University of Arizona, Tucson, AZ 85721, USA \\ ${ }^{2}$ Department of Biomedical Engineering, University of Arizona, Tucson, AZ 85721, USA \\ ${ }^{3}$ Department of Medical Imaging, University of Arizona, Tucson, AZ 85724, USA \\ ${ }^{4}$ University of Arizona Cancer Center, University of Arizona, Tucson, AZ 85724, USA
}

Correspondence should be addressed to Mark D. Pagel; mpagel@u.arizona.edu

Received 22 October 2014; Revised 8 February 2015; Accepted 8 February 2015

Academic Editor: Orazio Schillaci

Copyright (C) 2015 L. Q. Chen and M. D. Pagel. This is an open access article distributed under the Creative Commons Attribution License, which permits unrestricted use, distribution, and reproduction in any medium, provided the original work is properly cited.

\begin{abstract}
Tumor acidosis is a consequence of altered metabolism, which can lead to chemoresistance and can be a target of alkalinizing therapies. Noninvasive measurements of the extracellular $\mathrm{pH}(\mathrm{pHe})$ of the tumor microenvironment can improve diagnoses and treatment decisions. A variety of noninvasive imaging methods have been developed for measuring tumor pHe. This review provides a detailed description of the advantages and limitations of each method, providing many examples from previous research reports. A substantial emphasis is placed on methods that use MR spectroscopy and MR imaging, including recently developed methods that use chemical exchange saturation transfer MRI that combines some advantages of MR spectroscopy and imaging. Together, this review provides a comprehensive overview of methods for measuring tumor pHe, which may facilitate additional creative approaches in this research field.
\end{abstract}

\section{Introduction}

An emerging hallmark of cancer is the dysregulation of cellular energetics, which involves reprograming cellular energy metabolism to most effectively support neoplastic proliferation [1]. Under aerobic conditions, normal cells convert glucose to pyruvate via glycolysis in the cytoplasm and thereafter dispatch the pyruvate to the oxygen-consuming mitochondria to produce carbon dioxide and ATP (Figure 1) [2]. Under anaerobic conditions, pyruvate is converted to lactic acid in the cytosol. This anaerobic metabolism is inefficient as it produces $\sim 18$-fold fewer ATP molecules relative to mitochondrial oxidative phosphorylation. Warburg first reported that cancer cells limit their energy metabolism largely to glycolysis, even in the presence of oxygen [3]. Despite lower energy production efficiency, increased glycolysis allows the diversion of glycolytic intermediates into various biosynthetic pathways, including those generating nucleosides and amino acids [4]. This in turn facilitates the biosynthesis of the macromolecules and organelles required for assembling new cells, supporting the active cell proliferation in neoplastic disease.

The consequence of increased intracellular production of lactic acid is extracellular tumor acidosis. To maintain an intracellular $\mathrm{pH}(\mathrm{pHi})$ that is slightly alkaline $(\sim \mathrm{pH} 7.4)$, tumor cells upregulate several proton extrusion mechanisms such as the $\mathrm{Na}^{+} / \mathrm{H}^{+}$exchanger (NHE), $\mathrm{HCO}_{3}{ }^{-}$transporter, carbonic anhydrase IX, vacuolar-ATPase, and the $\mathrm{H}^{+} / \mathrm{K}^{+}$ ATPase $[5,6]$. Excess protons are excreted into the extracellular matrix, causing the extracellular $\mathrm{pH}(\mathrm{pHe})$ of the tumor microenvironment to become acidic. In certain tumor types such as human MCF-7 mammary carcinoma, the pHe has been measured to be as low as pH 6.44 [6]. Chronic exposure to acidic $\mathrm{pHe}$ has been reported to promote invasiveness and metastatic behavior in several tumor types [7-9].

Most chemotherapeutic agents enter cancer cells via passive diffusion across the cell membrane, which requires the agent to be in a nonionized form. Thus, the cell toxicities of weak-base drugs such as daunorubicin, doxorubicin, and mitoxantrone are greatly reduced under acidic conditions 


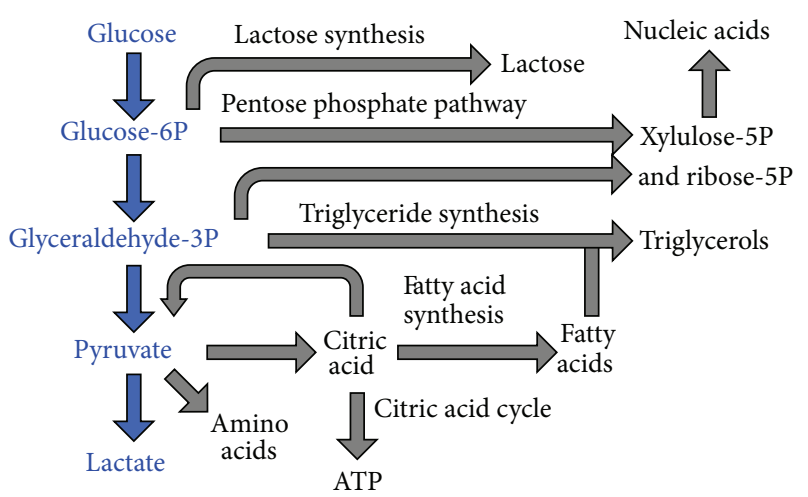

FIGURE 1: Schematic of glycolysis and associated metabolic pathways that create biomolecules for tumor growth. Adapted from [2].

[10-12] (Figure 2). When the $\mathrm{pH}$ drops below the $\mathrm{pKa}$ of these weak bases, they become predominantly protonated and positively charged and are therefore less permeable to cell membranes, resulting in cellular drug resistance. Conversely, weak acid chemotherapeutic drugs such as chlorambucil, cyclophosphamide, and 5-fluorouracil have higher cytotoxicity at lower $\mathrm{pH}[12]$. Hence, knowing the $\mathrm{pHe}$ of the tumor microenvironment can enable physicians to select the best chemotherapy based on tumor $\mathrm{pHe}$ and provide personalized chemotherapy for each individual patient.

Research has been conducted to investigate alkalinizing therapy as an option to increase tumor $\mathrm{pHe}$ that can enhance chemotherapy and counteract acid-mediated invasion and metastasis. In vitro results have shown that the cytotoxicity of doxorubicin has 2.25-fold enhancement when the $\mathrm{pH}$ of the cell culture media was raised from 6.8 to 7.4 [13]. In the same study, $200 \mathrm{mM}$ bicarbonate in drinking water was introduced ad libitum to a mouse model of MCF-7 mammary carcinoma. In vivo results showed that the tumor volume of the MCF-7 tumors in mice treated with both bicarbonate and doxorubicin were significantly smaller than mice treated with only doxorubicin $(P<0.03)$. In another study, bicarbonate treatment was also shown to reduce the formation of spontaneous metastases, with fewer metastatic lung lesions and longer survival times in mouse models of MDA-MB-231 metastatic breast cancer [14] (Figure 3). Computer simulations showed that the addition of a moderate amount of bicarbonate in blood that is $\sim 40 \%$ higher than normal serum concentration can reduce the amount of intratumoral and peritumoral acidosis and can almost completely eliminate tumor invasion [15]. Therefore, being able to accurately monitor pHe in tumors and normal tissues will greatly aid in evaluating the utility of alkalinizing treatment for cancer care.

\section{Imaging Methods to Measure In Vivo pH}

A variety of biomedical imaging methods have been developed in an attempt to measure tumor pHe (Table 1). Each method has shown a remarkable ability to produce quantitative measurements during in vivo studies, although the accuracies of these quantitative measurements have been difficult to confirm, due to a lack of a "gold standard". Perhaps more importantly, most of these methods have disadvantages which complicate or eliminate the possibility that the method can be translated to the radiology clinic to diagnose cancer patients. The following descriptions provide a summary of each method, including advantages and disadvantages.

\section{3. pH Measurements with Methods other than MRI}

3.1. $p$ H Electrode. The traditional ion-selective glass electrode is one of the first types of instrumentation that was used to measure $\mathrm{pH}$ in a human tumor. Although the use of an electrode is not an imaging method, $\mathrm{pH}$ measurements with an electrode are useful to compare to measurements made with noninvasive imaging methods. The $\mathrm{pH}$ of surface tumors such as malignant melanomatosis can be measured using a $\mathrm{pH}$ electrode because these tumors are easily accessible to the percutaneous technique [16]. At the completion of each procedure, the nodule has to be excised to avoid the possibility of tumor fungating. More recently, a needle-shaped $\mathrm{pH}$ electrode has been developed to minimize the invasiveness and improve the speed of this technique (Figure 4). The $\mathrm{pH}$ measurement is assumed to be accurate because this microelectrode can be accurately calibrated with external buffer solutions. The spatial resolution depends on the number of insertions and the spacing between each insertion into the solid tumor, which has ranged between 4 and 20 insertions at $0.5-1.0 \mathrm{~cm}$ intervals in tumor studies with canines [17]. When the tumor is not on the surface, an additional localizer step such as MRI is required to locate the tumor and assist the placement of the $\mathrm{pH}$ electrode into the tumor and to direct the electrodes away from necrotic areas in the tumor. Due to inevitable tissue damage during electrode insertion into tissue, the measured $\mathrm{pH}$ must be a weighted average of $\mathrm{pHi}$ and $\mathrm{pHe}$ and may also be affected by potential damage to microvasculature [18].

3.2. Optical Imaging with Fluorescence. Optical imaging with a fluorescence dye is a low cost imaging tool with great versatility. A fluorescence agent can be engineered to couple with a peptide, antibody, or other biomolecule, which can bind to a tumor biomarker and enhance selectivity for tumor tissue [25]. The $\mathrm{pH}$ can be measured independent of concentration by assessing the ratio of fluorescence signals at different emission wavelengths (Figure 5) [19-21, 26] or at different fluorescence lifetimes [27]. Near-infrared (NIR) light at $700-900 \mathrm{~nm}$ wavelength is preferred for in vivo imaging because NIR light can propagate through tissue to a depth of approximately one centimeter and still be adequately detected relative to background signals, due to lower tissue absorption and autofluorescence in this wavelength regime [25]. Optical imaging has high sensitivity and can detect nanomolar concentrations of agents. It also has good temporal resolution on the order of seconds [14, 26, 28, 29]. Surface-accessible tumors can be imaged at excellent micronscale spatial resolution. Based on these merits, preclinical optical imaging has been applied to surface-accessible tumors 


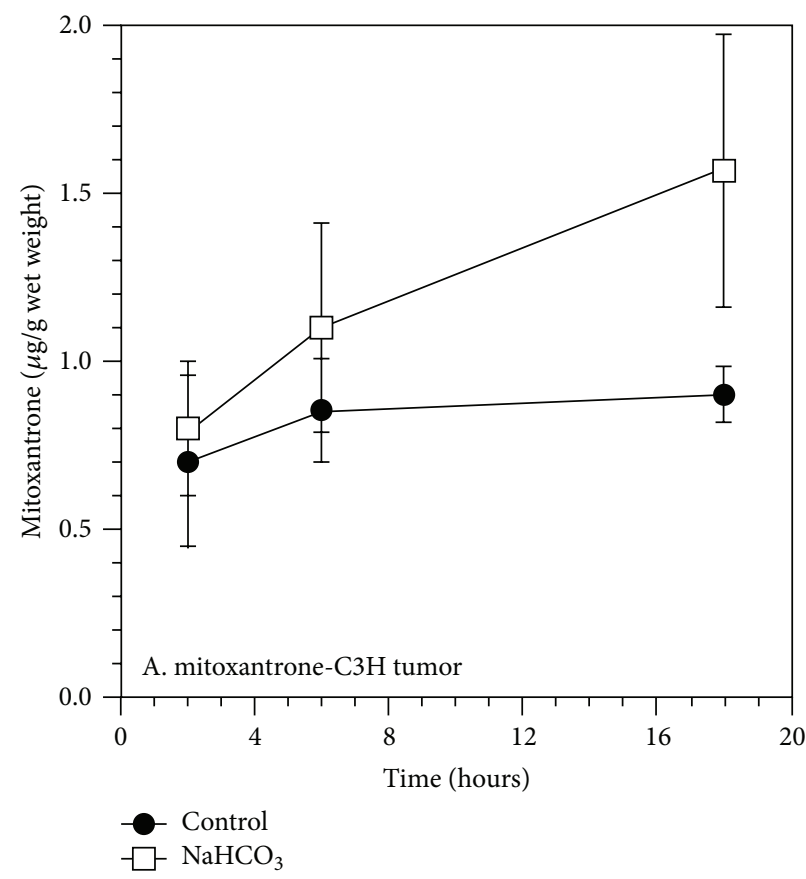

(a)

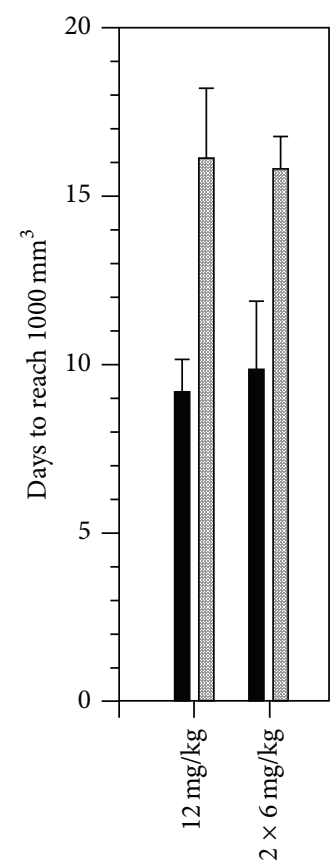

(b)

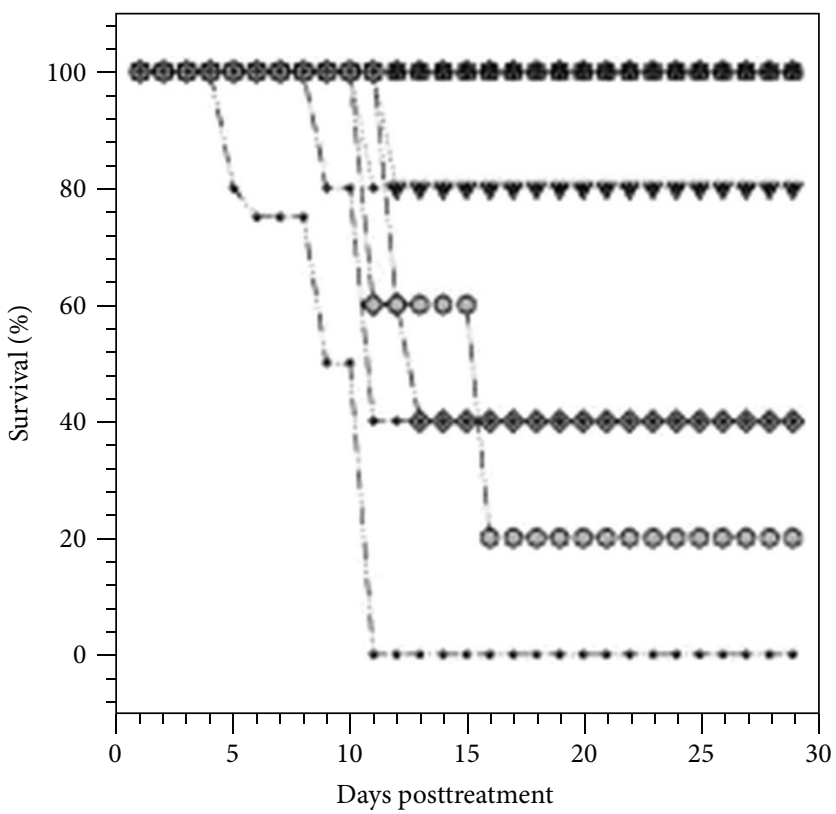

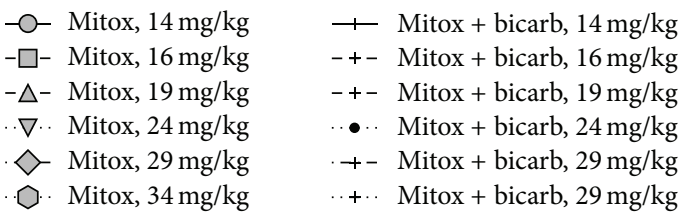

(c)

FIGURE 2: Tumor acidosis causes chemoresistance against weak-base drugs. (a) Uptake and retention of mitoxantrone, a weak-base drug, was greater in $\mathrm{C} 3 \mathrm{H}$ tumor tissue that was neutralized with sodium bicarbonate. (b) Mitoxantrone treatment (1 dose of $12 \mathrm{mg} / \mathrm{kg}$ or $2 \mathrm{doses}$ of $6 \mathrm{mg} / \mathrm{kg}$ ) caused a greater growth delay when sodium bicarbonate neutralized the tumor acidosis. (c) Sodium bicarbonate also improved survival with mitoxantrone treatment. Reproduced with permission from [10]. 
TABLE 1: Summary of $\mathrm{pH}$ measurement methods with various instruments.

\begin{tabular}{|c|c|c|c|}
\hline Method & $\begin{array}{l}\text { Sampling time } \\
\text { Spatial resolution } \\
\text { Agent }[C]\end{array}$ & $\begin{array}{l}\text { Advantages } \\
\text { and } \\
\text { disadvantages }\end{array}$ & Reference \\
\hline Microelectrode & $\begin{array}{l}\text { Fast, sec } \\
\text { Localized measurement } \\
\text { No agent required }\end{array}$ & $\begin{array}{l}\text { Fast; accurate if calibrated with an external buffer } \\
\text { Limited to surface-accessible tumors; requires MRI to guide electrodes into } \\
\text { tumors }\end{array}$ & [16-18] \\
\hline $\begin{array}{l}\text { Fluorescence } \\
\text { imaging }\end{array}$ & $\begin{array}{l}\text { Fast, sec } \\
5 \mu \mathrm{m} \\
\mu \mathrm{M}-\mathrm{nM}\end{array}$ & $\begin{array}{l}\text { Sensitive; low cost; can be used during clinical fluorescence guided surgery } \\
\text { Limited to surface-accessible tumors }\end{array}$ & $\begin{array}{l}{[14,19-} \\
21,25-29]\end{array}$ \\
\hline PET & $\begin{array}{l}10 \mathrm{~min} \\
2 \mathrm{~mm} \\
\mathrm{nM}\end{array}$ & $\begin{array}{l}\text { Fast; whole body imaging } \\
\text { Requires radioactive isotope; coarse resolution; and limited accuracy }\end{array}$ & {$[22-24,31]$} \\
\hline${ }^{1} \mathrm{H}$ MRS & $\begin{array}{l}>30 \mathrm{~min}^{3} \\
1 \mathrm{~mm}^{3} \\
\mathrm{mM}\end{array}$ & $\begin{array}{l}\text { Simultaneous measurement of } \mathrm{pHe} \text { and detection of metabolites } \\
\text { Poor sensitivity; some agents are } \mathrm{pH} \text { buffers that change tissue } \mathrm{pHe}\end{array}$ & {$[34,39,40]$} \\
\hline${ }^{31} \mathrm{P}$ MRS & $\begin{array}{l}40 \mathrm{~min}^{3} \\
1 \mathrm{~cm}^{3} \\
\mathrm{mM}\end{array}$ & $\begin{array}{l}\text { Can simultaneously measure } \mathrm{pHi} \text { and } \mathrm{pHe} \\
\text { Requires } a^{31} P \text { MRI transceiver coil }\end{array}$ & {$[41,43-46]$} \\
\hline${ }^{19} \mathrm{~F}$ MRS & $\begin{array}{l}5 \mathrm{~min}^{3} \\
1 \mathrm{~cm}^{3} \\
\mu \mathrm{M}-\mathrm{mM}\end{array}$ & $\begin{array}{l}\text { Fast; good sensitivity } \\
\text { Requires } a{ }^{19} \text { F MRI transceiver coil }\end{array}$ & {$[42,47,49]$} \\
\hline $\begin{array}{l}\text { Hyperpolarized } \\
{ }^{13} \mathrm{C} \text { MRS }\end{array}$ & $\begin{array}{l}5 \mathrm{sec} \\
0.375 \mathrm{~mm}^{3} \\
\mathrm{mM}\end{array}$ & $\begin{array}{l}\text { Very fast } \\
\text { Requires }{ }^{13} \mathrm{C} M R I \text { transceiver coil; requires a hyperpolarizer instrument } \\
\text { Short hyperpolarized }{ }^{13} \mathrm{C} \text { life time; } \\
\text { Measures pHi and pHe }\end{array}$ & {$[48]$} \\
\hline $\begin{array}{l}\mathrm{pH} \text { dependent } \\
T_{1} \text { relaxation }\end{array}$ & $\begin{array}{l}<1 \mathrm{~min}^{3} \\
0.1 \mathrm{~mm}^{3} \\
\mathrm{mM}\end{array}$ & $\begin{array}{l}\text { Fast; high resolution } \\
\text { Requires a cocktail of contrast agents }\end{array}$ & [50-53] \\
\hline CEST MRI & $\begin{array}{l}\sim 5 \mathrm{~min} \\
\mu \mathrm{m}-\mathrm{mm} \\
\mathrm{mM}\end{array}$ & $\begin{array}{l}\text { Good specificity } \\
\text { Poor sensitivity }\end{array}$ & {$[37]$} \\
\hline
\end{tabular}
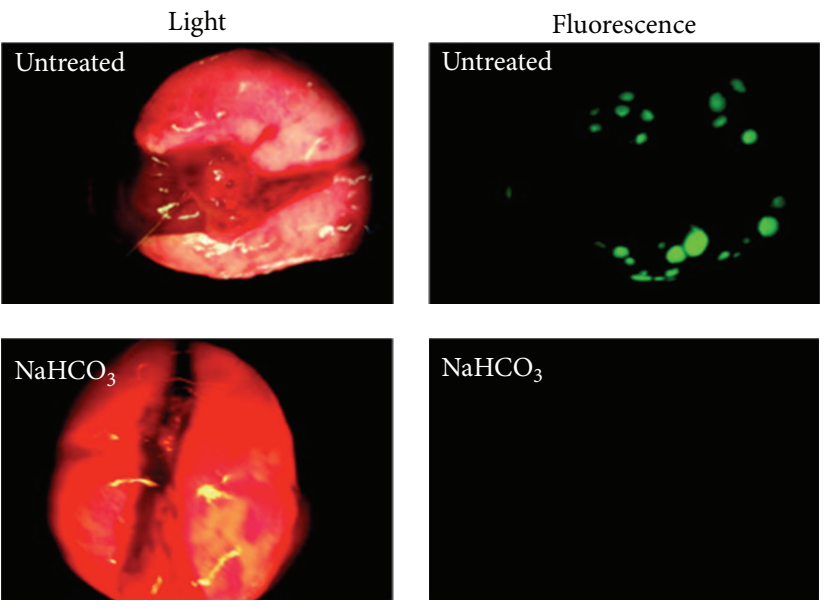

(a)

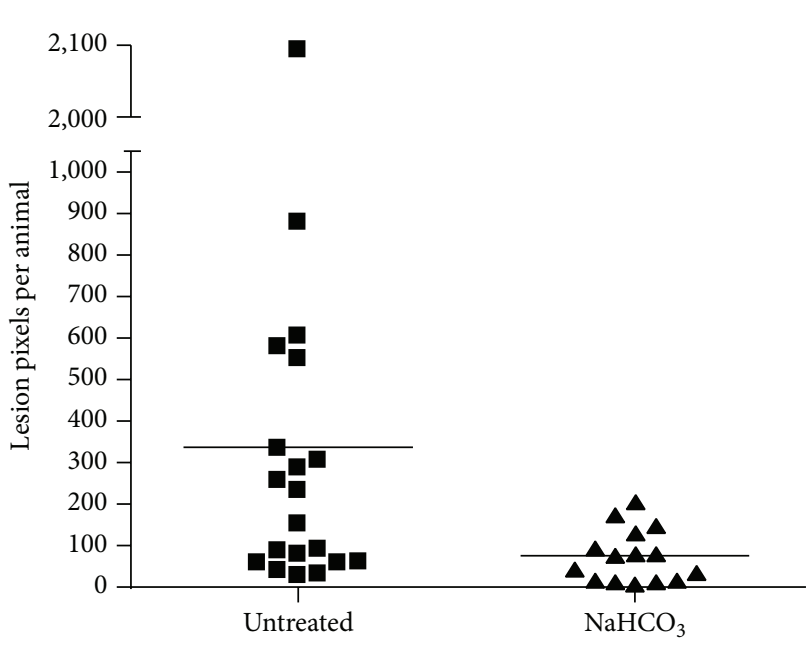

(b)

FIGURE 3: Treatment of a mouse model of $\beta$-galactosidase-labeled MDA-MB-231 mammary carcinoma with $200 \mathrm{mM} \mathrm{NaHCO}_{3}$ ad libitum for 60 days resulted in lower lung metastases as evidenced by (a) $\beta$-galactosidase-induced fluorescence staining of lung lesions and (b) the number of lesion pixels per animal. Reproduced with permission from [14]. 


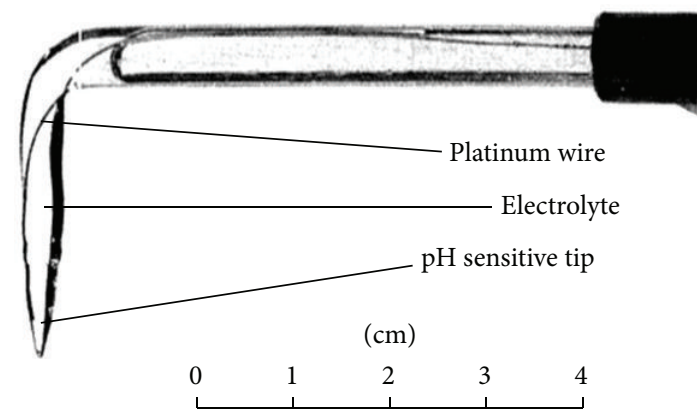

(a)

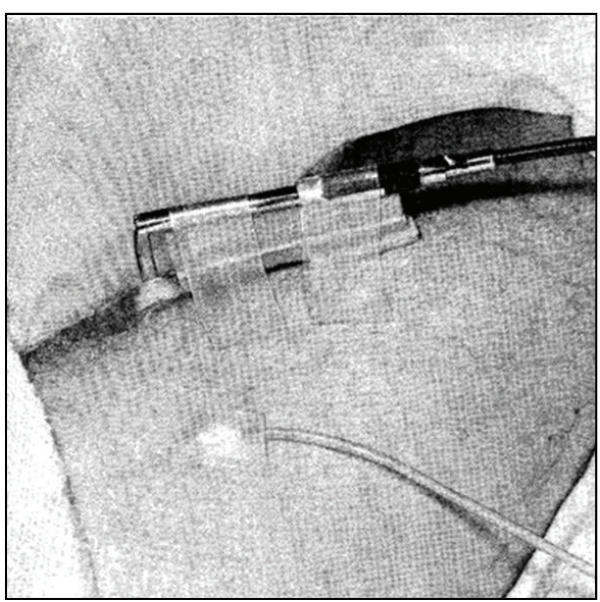

(b)

FIGURE 4: A pH microelectrode for measuring in vivo tumor $\mathrm{pH}$. (a) A photo of an angled glass electrode with a platinum wire and $0.1 \mathrm{~N}$ hydrochloric acid electrolyte. (b) The microelectrode was inserted into a melanoma nodule of a patient, stabilized with sponge rubber between the skin and electrode shaft, and secured with adhesive tape. A calomel reference electrode was secured to the skin. Adapted from [16] with permission.

such as the rabbit ear chamber [29] and window chamber models of mammary carcinoma [14], and optical imaging has been used during image-guided surgery that provides access to the surface of tumors [30]. However, optical imaging cannot interrogate tumors that are located in deep tissues, which severely limits the utility of this imaging method for measuring tumor pHe. Furthermore, lower optical signals generated from deeper regions of surface-accessible tumors can weigh the measurement to the characteristics of the tumor surface. For these reasons, pHe measurements with optical imaging have not been translated to routine clinical use.

\subsection{Positron Emission Tomography. Positron emission to-} mography (PET) is a widely used molecular imaging modality in both clinical and research settings. Whole-body PET imaging of mice can be performed in less than 10 minutes. To image tumor $\mathrm{pHe}$ with $\mathrm{PET}$, a ${ }^{64} \mathrm{Cu}$ radioactive nuclide has been conjugated to the $\mathrm{pH}$ low insertion peptide (pHLIP), which folds to form an $\alpha$-helix and inserts itself into cell membranes when the tumor pHe is acidic (Figure 6) [22-24, 31]. This method has high sensitivity and therefore can produce images with an administration of agent as low as $\sim 0.01 \mathrm{ng} / \mathrm{kg}$ of mouse weight. Higher retention of the agent within the tumor has been shown to correlate with lower acidity [22]. However, this method depends on an equilibrium between the peptide conformations that can and cannot insert into a cell membrane, and therefore the fraction of membrane-inserted peptide has a sigmoidal relationship with $\mathrm{pH}$. This causes the $\mathrm{pH}$ measurement to be semiqualitative instead of quantitative. Furthermore, PET has spatial resolution of $\sim 2 \mathrm{~mm}$ [32] and may not be as applicable to measure $\mathrm{pH}$ in small tumors such as tumors in lymph nodes and may not be able to adequately assess tumor heterogeneity.
3.4. Electron Paramagnetic Resonance. Electron paramagnetic resonance (EPR) spectroscopy is a technique for studying material with unpaired electrons. Because most stable molecules have all of their electrons paired, only a few molecules are sensitive to EPR. This limitation also means that EPR offers great specificity and there is no competing background signal [35]. The use of $\mathrm{pH}$-sensitive nitroxides offers a unique opportunity for noninvasive assessment of $\mathrm{pH}$ values from 0 to 14 in living animals with sensitivity in the $\mu \mathrm{M}$ regime [36]. The $\mathrm{pH}$ can be determined by measuring the frequency separation of the spectral peaks or by quenching the EPR signal within a $\mathrm{pH}$-dependent polymer (Figure 7) [33]. Unfortunately, tissues must be irradiated with high power to perform EPR studies, which limits this technique to the study of small animal models and peripheral human tissues.

\section{Magnetic Resonance Based Methods other than CEST MRI}

Magnetic resonance imaging (MRI) and MR spectroscopy (MRS) are excellent whole-body imaging tools that provide excellent soft tissue contrast with little radiation exposure. Beyond anatomical information, recent MRI and MRS developments have focused on providing environmental biomarker evaluations ( $\mathrm{pH}$, temperature, and oxygen) and molecular information (proteins, enzyme activity, gene expression, metabolites, and metal ions) [37]. In recent years, innovations in MR instrumentation have drastically improved spatial and temporal resolution, and it is now possible to image in vivo tissues with $\sim 0.1 \mathrm{~mm}$ spatial resolution.

4.1. Magnetic Resonance Spectroscopy. Magnetic resonance spectroscopy (MRS) has been used for more than three decades to examine metabolite distributions among living 


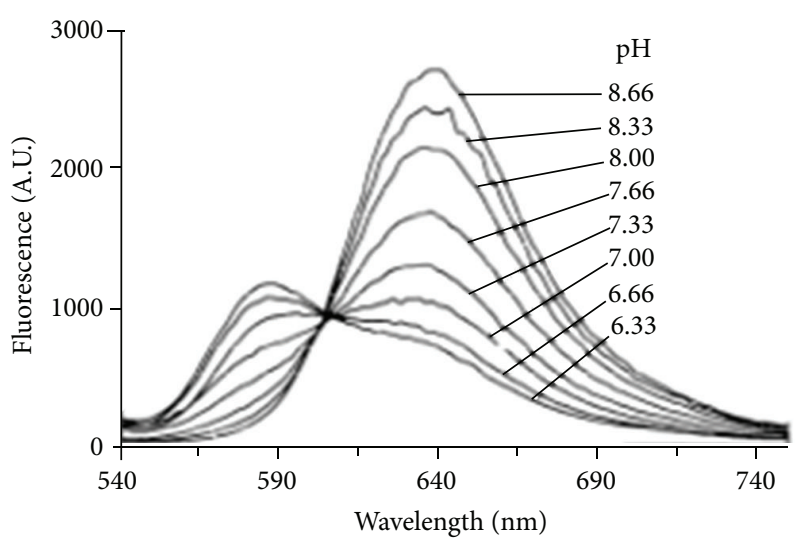

(a)

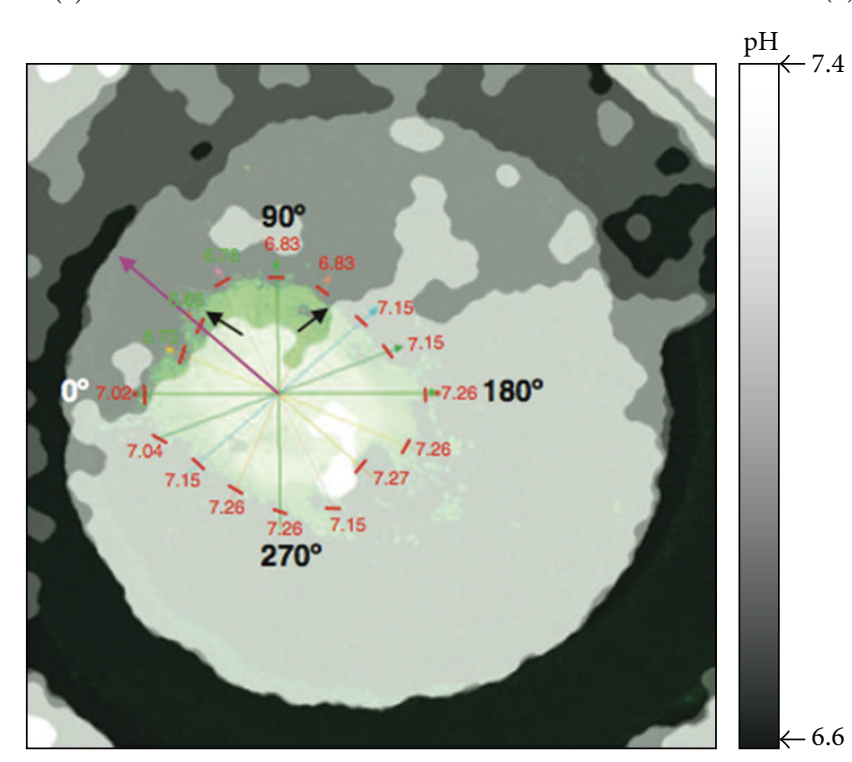

(c)

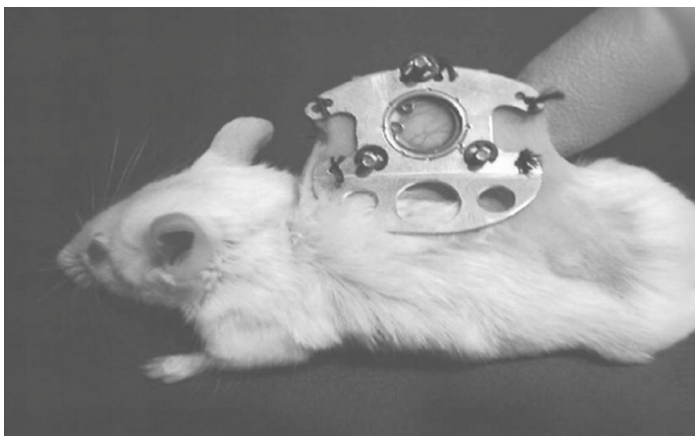

(b)

FIgURE 5: Fluorescence imaging of tumor pHe. (a) The emission spectrum of SNARF-1 dye shows that pH is correlated with the ratio of the fluorescence signal at 570/650 nm. (b) A window chamber model can access a solid tumor growing under a coverslip in a skinfold. (c) The parametric map of pHe was determined from the ratio of fluorescence signals of SNARF-1 dye in an in vivo window chamber model of a HCT116-GFP tumor. Black and purple arrows indicate acidic environment toward which the tumor is growing. The pHe values of the tumor edge are listed near short red lines. Reproduced with permission from [19-21].

cells and tissues. The most common applications of in vivo MRS are used to detect endogenous signals from ${ }^{1} \mathrm{H},{ }^{31} \mathrm{P}$, or ${ }^{23} \mathrm{Na}$ [38]. ${ }^{1} \mathrm{H}$ MRS is the most favorable spectroscopic method because ${ }^{1} \mathrm{H}$ has the highest inherent sensitivity with 99.98\% natural abundance and the highest gyromagnetic ratio of stable isotopes [39], and most clinical MR instruments have the capability to detect ${ }^{1} \mathrm{H}$ MR signals. Furthermore, the most atoms in the human body are hydrogen atoms, $67 \%$ by atomic percent. Tumor pHe can be measured by detecting endogenous metabolites that have chemical shifts that are sensitive to $\mathrm{pH}$. For example, $\mathrm{pH}$ is correlated with the chemical shift difference between the two protons on histidine, both of which are $\mathrm{pH}$-sensitive. Such analyses do not require known concentrations of histidine [40]. Another example is $( \pm) 2$-imidazole-1-yl-3ethoxycarbonylpropionic acid (IEPA), which can measure
$\mathrm{pH}$ by comparing the $\mathrm{pH}$-dependent chemical shift of one proton with the $\mathrm{pH}$-independent chemical shift of a second proton in the same molecule (Figure 8) [34]. Thus a ratio of the two chemical shifts correlates with $\mathrm{pH}$ in a concentration independent manner. However, the use of IEPA to measure $\mathrm{pHe}$ is questionable, because IEPA is a $\mathrm{pH}$ buffer and can alter the $\mathrm{pHe}$ by interfering with the clearance of extracellular proton to the blood stream.

${ }^{31} \mathrm{P}$ MRS has been used to measure the chemical shift of endogenous inorganic phosphate $\left(\mathrm{P}_{\mathrm{i}}\right)$. However, this chemical shift is weighted to reporting the pHi rather than the $\mathrm{pHe}$, because $\sim 2-3 \mathrm{mM}$ of $\mathrm{P}_{\mathrm{i}}$ is in the intracellular compartment and only $\sim 1 \mathrm{mM}$ is in the extracellular compartment [39]. The exogenous contrast agent 3-aminopropylphosphonate (3-APP) can be used to measure $\mathrm{pHe}$ via ${ }^{31} \mathrm{P}$ MRS (Figure 9) [41]. The chemical shift of 3-APP is referenced to the chemical 


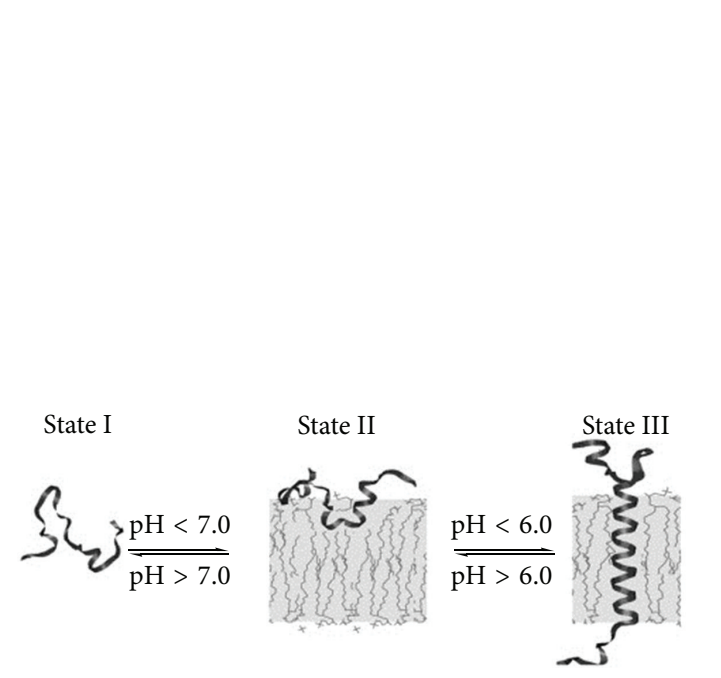

(a)
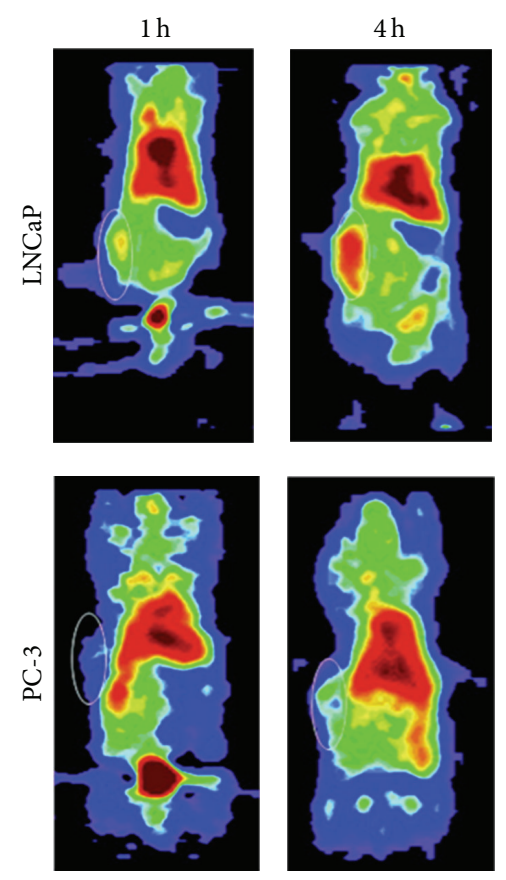

(c)

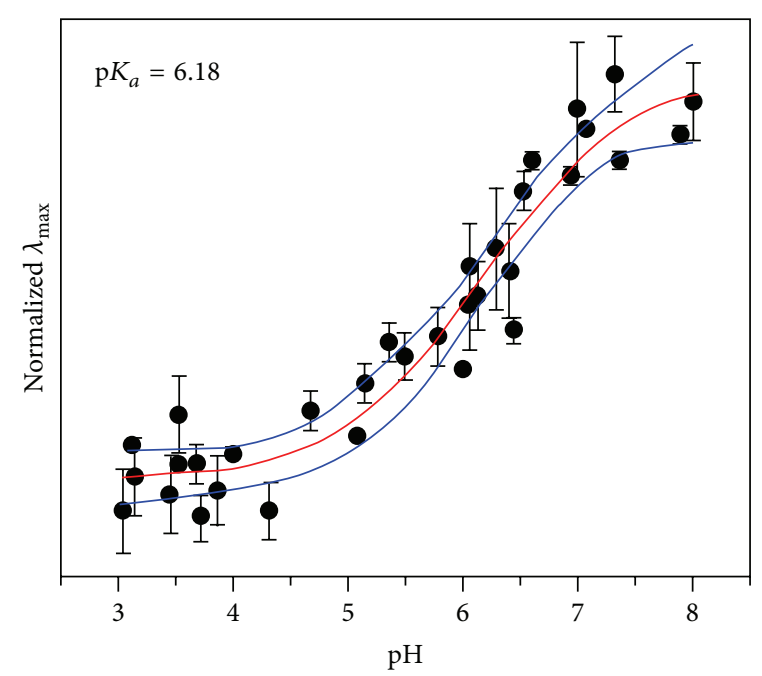

(b)
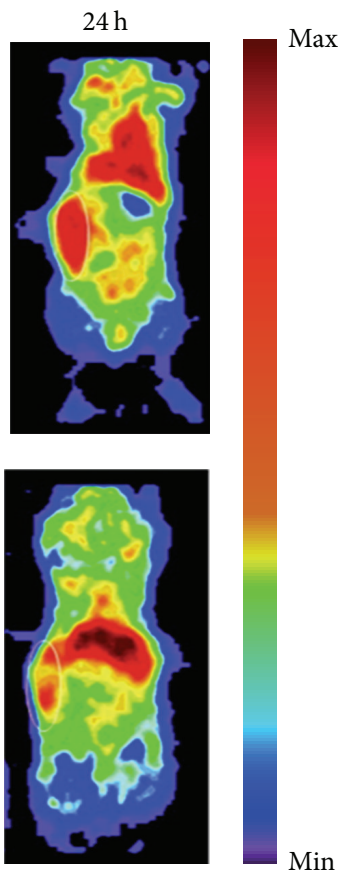

FIGURE 6: PET imaging of tumor pHe. (a) A pHLIP peptide inserts into cell membranes in low pHe conditions. (b) PET imaging of ${ }^{64} \mathrm{Cu}-$ DOTA-pHLIP showed higher uptake and retention in a subcutaneous mouse model of LNCaP relative to PC-3, indicating that the LNCaP tumor model had lower pHe. MR spectroscopy confirmed that the average pHe values of the LNCap and PC-3 tumor models were $6.78 \pm$ 0.29 and $7.23 \pm 0.10$, respectively. White circles show the locations of the subcutaneous tumors. (c) A membrane-insertion of a variation of pHLIP shows a sigmoidal dependence on $\mathrm{pH}$. Reproduced with permission from [22-24].

shift of the alpha peak of APT or GPC and therefore information about the concentration of 3-APP is not required. Furthermore, 3-APP has low toxicity and can measure pHe within a physiological range of $\mathrm{pH} 6$ to 8 . This method of analysis allows simultaneous $\mathrm{pHe}$ and $\mathrm{pHi}$ measurements with 3-APP and $\mathrm{P}_{\mathrm{i}}[43,44]$ and has been used to study alkalosis and acidification of tumor models $[45,46]$. This method has been a popular choice as a cross-reference when developing new methods to measure $\mathrm{pHe}[22,47,48]$.
Even though ${ }^{31} \mathrm{P}$ MRI has $100 \%$ natural abundance, its signal sensitivity relative to ${ }^{1} \mathrm{H}$ MRI signals is only $6.6 \%$. For comparison, ${ }^{19} \mathrm{~F}$ is $100 \%$ naturally abundant and has a MR sensitivity of $83 \%$ relative to ${ }^{1} \mathrm{H}$ [39]. Furthermore ${ }^{19} \mathrm{~F}$ MRS has a relatively large chemical shift difference and an almost lack of endogenous MR signal in normal tissues. The fluorinated compound ZK-150471 emits two ${ }^{19} \mathrm{~F}$ MR signals that have a frequency difference which is dependent on $\mathrm{pH}$ (Figure 10) [42]. This ${ }^{19} \mathrm{~F}$ agent has been used to 


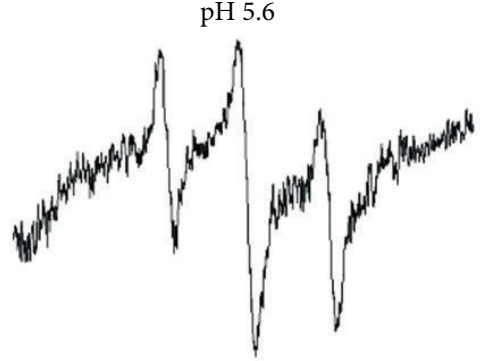

(a)

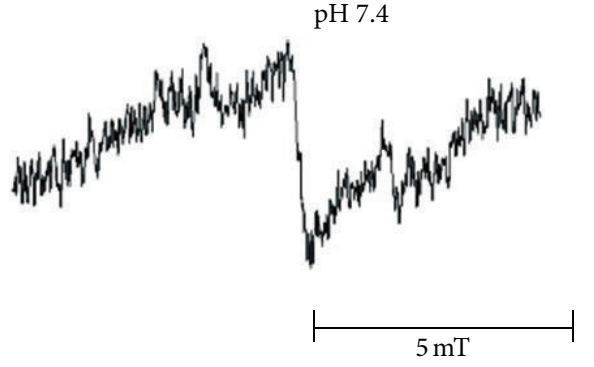

(b)

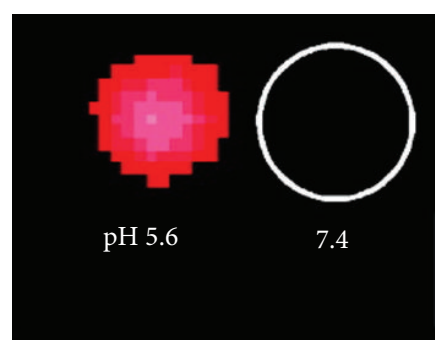

(c)

FIGURE 7: EPR imaging of pH. The nitroxide radical TEMPO shows strong EPR signals in solution, which are quenched when TEMPO is encapsulated in a nanoparticle. (a) Degradation of the nanoparticle at low $\mathrm{pH}$ dequenches the EPR signals from TEMPO. (b) No change in the nanoparticle at neutral $\mathrm{pH}$ retains the EPR-quenched state. (c) Phantom images demonstrate that the difference in $\mathrm{pH}$ can be spatially localized. Reproduced with permission from [33].

detect changes in tumor pHe in response to treatment with hydralazine and/or heating. In a study that compared ${ }^{19} \mathrm{~F}$ and ${ }^{31} \mathrm{P}$ MRS at a $1 \mathrm{~cm}^{3}$ spatial resolution, ${ }^{31} \mathrm{P}$ MRS required $\sim 40$ min of acquisition time, while its counterpart ${ }^{19} \mathrm{~F}$ MRS only required $\sim 5 \mathrm{~min}$ [47]. However, $\mathrm{pH}$-sensitive ${ }^{19} \mathrm{~F}$ contrast agents have practical problems that limit in vivo use, such as the instability of some fluorinated compounds and nonspecific accumulation in normal tissues, which can result in low sensitivity in the tumor tissue. To improve stability and specificity, ${ }^{19} \mathrm{~F}$ contrast agents can be encapsulated into nanogels that specifically target a tumor. The diameter of the nanogel is $\mathrm{pH}$-sensitive, and indirectly measuring a change in diameter via ${ }^{19} \mathrm{~F}$ MRS provides a $\mathrm{pH}$ measurement [49]. In addition, the accuracy of pHe measurements with ${ }^{19} \mathrm{~F}$ agents may be questionable, partly due to many potential biomolecular interactions with the agent that can change the chemical shift of the ${ }^{19} \mathrm{~F}$ nucleus.

Despite the promise of ${ }^{31} \mathrm{P}$ and ${ }^{19} \mathrm{~F}$ MRS for measuring tumor pHe, these MRS methods have many detriments for clinical use. Most clinical MRI instruments do not have the capability to measure isotopes other than ${ }^{1} \mathrm{H}$. A typical ${ }^{1} \mathrm{H}$ MRS result requires a long acquisition time greater than $30 \mathrm{~min}$ and provides only coarse resolution of approximately $1 \mathrm{~cm}^{3}$. Due to the low signal to noise ratio (SNR), MRS requires a high degree of magnetic field homogeneity and minimal movement [39]. Over the years, strategies have been developed to reduce acquisition time and/or to increase spatial resolution. For example, point resolved spectroscopy (PRESS) [69] and stimulated echo acquisition mode (STEAM) [70] have been developed to enable simultaneous acquisition of spectra from multiple volumes at $1 \mathrm{~mm}^{3}$ spatial resolution. However, the relationship between SNR, spatial resolution, and acquisition time are dictated by the physics of the nuclear spins and electronics. Hence, improved resolution or reduced acquisition time is always associated with reductions in SNR. For example, a $40 \mathrm{~mL}$ voxel interrogated with a clinical $1.5 \mathrm{~T}$ MRI instrument requires $30 \mathrm{~min}$ acquisition time, while a $105 \mathrm{~mL}$ voxel requires only $6 \mathrm{~min}$ of acquisition time with a $1.5 \mathrm{~T}$ MRI instrument [40].
4.2. Hyperpolarized ${ }^{13} \mathrm{C}$ Spectroscopy. The ${ }^{13} \mathrm{C}$ isotope has $1.1 \%$ natural abundance and a MRS sensitivity of $0.016 \%$ relative to ${ }^{1} \mathrm{H}$ MRS at $37^{\circ} \mathrm{C}$. Dynamic nuclear polarization (DNP) can be used to increase the sensitivity of ${ }^{13} \mathrm{C}$ MRS [71]. This technique involves cooling a ${ }^{13} \mathrm{C}$ labeled molecule to $\sim 1 \mathrm{~K}$ and then transferring polarization from electron spins to ${ }^{13} \mathrm{C}$ nuclei with microwave irradiation. The sample is then warmed rapidly to body temperature while retaining a high level of nuclear spin polarization. A ratio of $\mathrm{H}^{13} \mathrm{CO}_{3}{ }^{-}$and ${ }^{13} \mathrm{CO}_{2}$ from injected ${ }^{13} \mathrm{C}$ labeled bicarbonate is correlated with $\mathrm{pH}$ (Figure 11) [48]. Each acquisition is $\sim 5 \mathrm{sec}$ and yields a resolution of $0.375 \mathrm{~mm}^{3}$ per voxel. Despite the ultrafast acquisition, hyperpolarized ${ }^{13} \mathrm{C}$ has a rapid decay of $\sim 20 \mathrm{sec}$ and requires a special transceiver coil and hyperpolarizer. Furthermore, ${ }^{13} \mathrm{C}$ labeled bicarbonate can only measure a weighted average of $\mathrm{pHi}$ and $\mathrm{pHe}$.

4.3. $T_{1}$ Relaxivity MRI. The use of a paramagnetic metal complex as a MRI contrast agent is now widely accepted in diagnostic radiology. Agents currently approved for clinical use are based on low-molecular weight chelates of gadolinium that partition throughout all extracellular space and enhance the $T_{1}$ MR relaxation of nearby water protons. The $T_{1}$ relaxivities of some MRI contrast agents are $\mathrm{pH}$-dependent, such as $\mathrm{Gd}(\mathrm{III})$ chelates that have a $\mathrm{pH}$-sensitive ligand, which can block water from accessing the Gd(III) ion only under certain $\mathrm{pH}$ conditions [37, 72-80]. Other agents currently approved for clinical use are based on superparamagnetic iron oxide nanoparticles that enhance the $T_{2} \mathrm{MR}$ relaxation of nearby water protons. For example, iron oxide nanoparticles encapsulated within $\mathrm{pH}$-responsive nanocapsules [81] and $\mathrm{pH}$-sensitive hydrogels [82] exhibit different $T_{2}$ relaxation properties under different $\mathrm{pH}$ conditions.

Among these relaxation-based MRI contrast agents, GdDOTA-4Amp has been applied to measure in vivo $\mathrm{pHe}$ with the greatest success [85]. Knowledge on the concentration of Gd-DOTA-4Amp is required to convert the $T_{1}$-weighted MRI signal to a pHe measurement, because the MRI signal is dependent on both concentration and the $T_{1}$ relaxation 


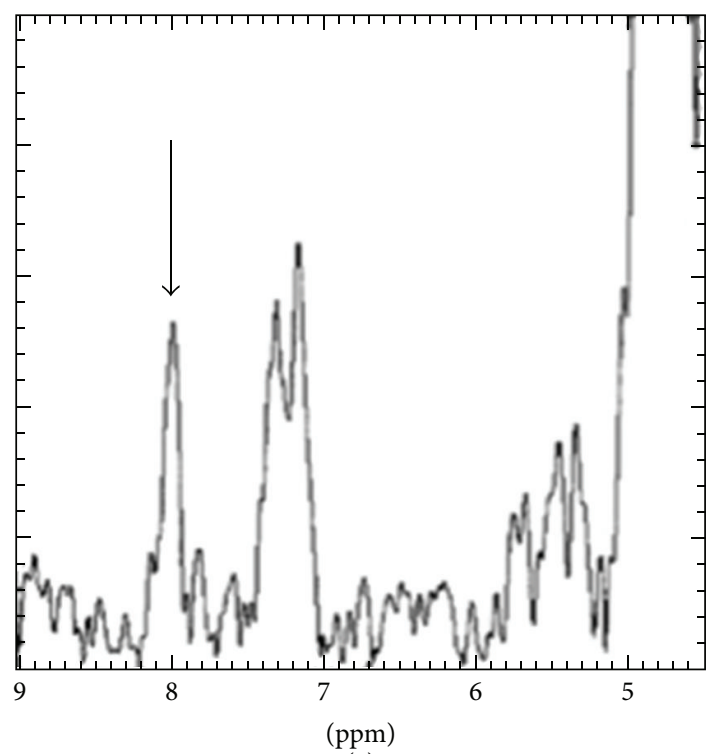

(a)

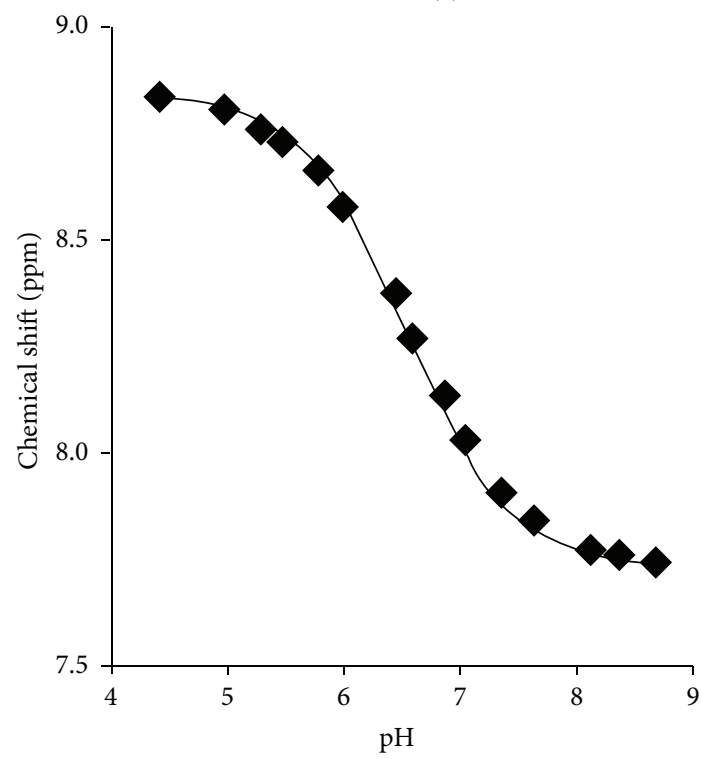

(b)

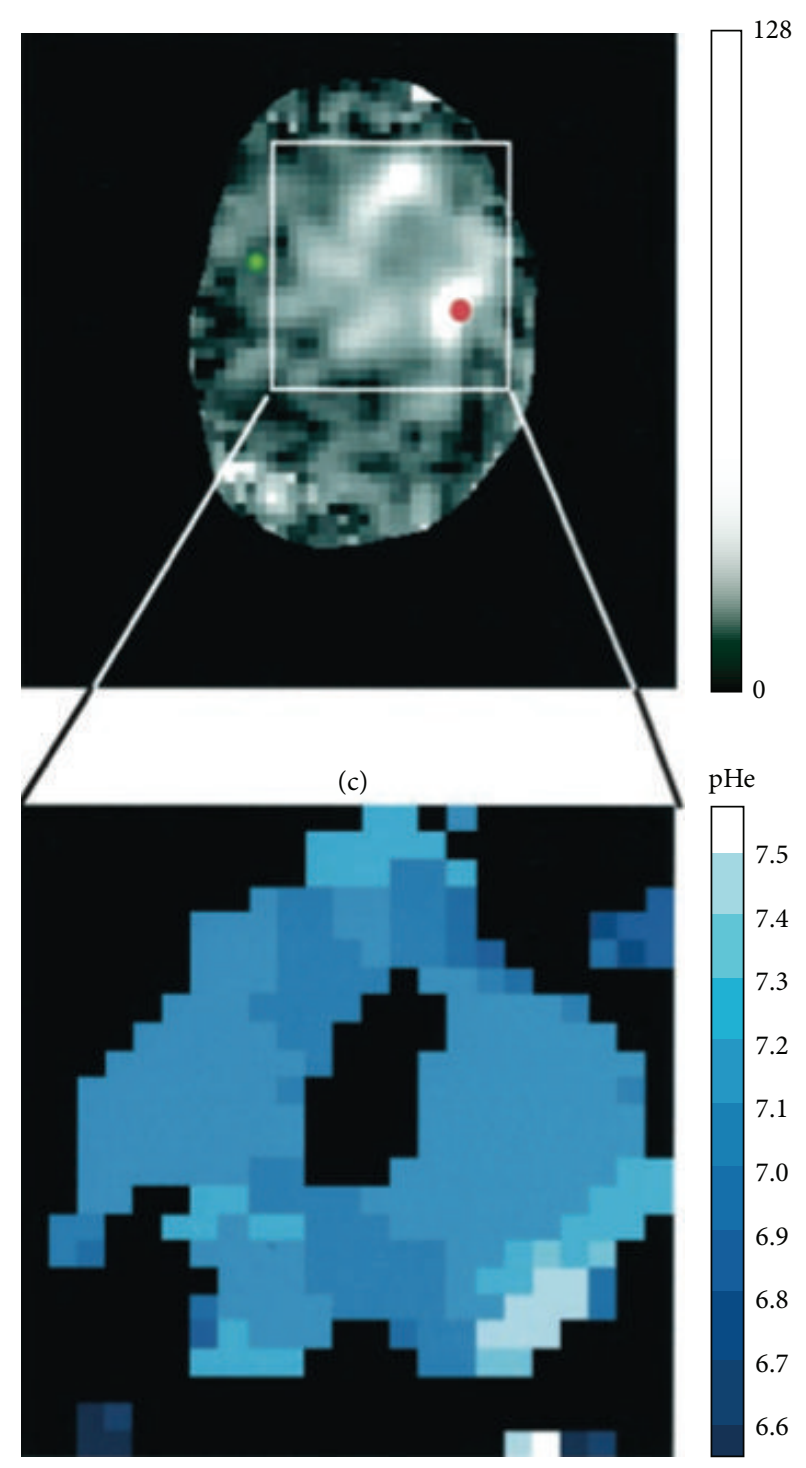

(d)

FIGURE 8: ${ }^{1} \mathrm{H}$ MR spectroscopic imaging of tumor pHe. (a) The ${ }^{1} \mathrm{H}$ spectrum from within a glioma showed the chemical shift of the IEPA H2 resonance (arrow), (b) which is correlated with $\mathrm{pH}$ as shown by an in vitro titration. (c) A parametric map of IEPA signal amplitudes showed accumulation of the agent in the glioma. (d) A parametric map of pHe was determined from the chemical shift of the IEPA H2 resonance and the correlation shown in (b). Reproduced with permission from [34].

effect of the agent. A pH-unresponsive contrast agent with an analogous chemical structure can be used to account for the concentration of $\mathrm{pH}$-responsive agent [37]. For example, two serial boluses of Gd-DOTA-4AmP and Gd-DOTP have been used to measure tumor pHe in $\mathrm{C} 6$ glioma and renal carcinoma [50-52]. The $T_{1}$-weighted MRI signal from GdDOTP was used to determine the temporally dynamic concentration of this agent in the tumor, which was assumed to be identical to the temporally dynamic concentration of GdDOTA-4AmP. Based on this concentration, the $T_{1}$-weighted MRI signal of Gd-DOTA-4AmP was used to determine the pHe. However, this method with serial injections makes the risky assumption that the biodistributions are identical for two MRI contrast agents that are administered at different times. To avoid this assumption, a single cocktail of GdDOTA-4AmP and Dy-DOTP has been administered to a mouse model of C6 glioma (Figure 12) [53]. The Gd-DOTA4Amp dominated the $T_{1}$ relaxation process generated by the agents, whereas Dy-DOTP dominated the $T_{2}{ }^{*}$ relaxation process of the agents. The $T_{2}{ }^{*}$ relaxation effect was then used to determine the pixelwise concentration of Dy(III), which was assumed to be identical to Gd(III). This value was used to convert the pixelwise $T_{1}$-weighted MRI signals into $\mathrm{pH}_{\mathrm{e}}$ maps. In a similar in vitro study, the ratio of $T_{1}$ and 


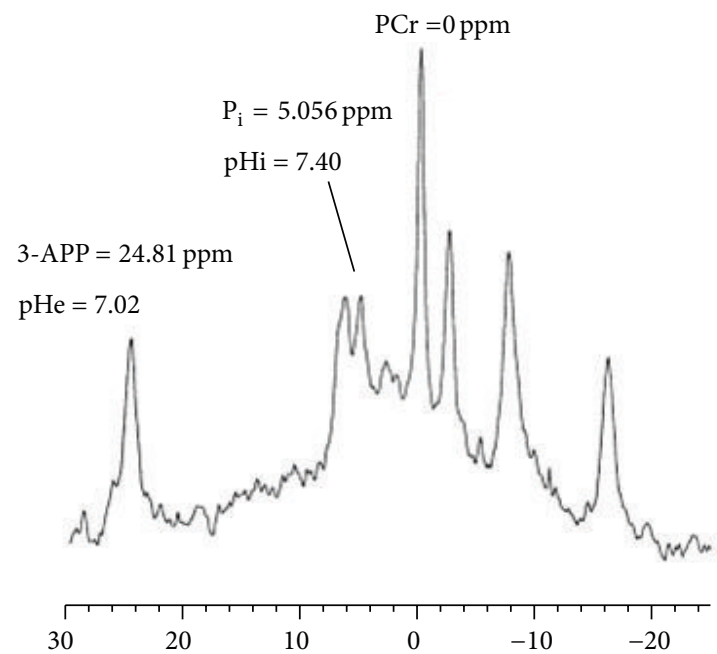

(a)

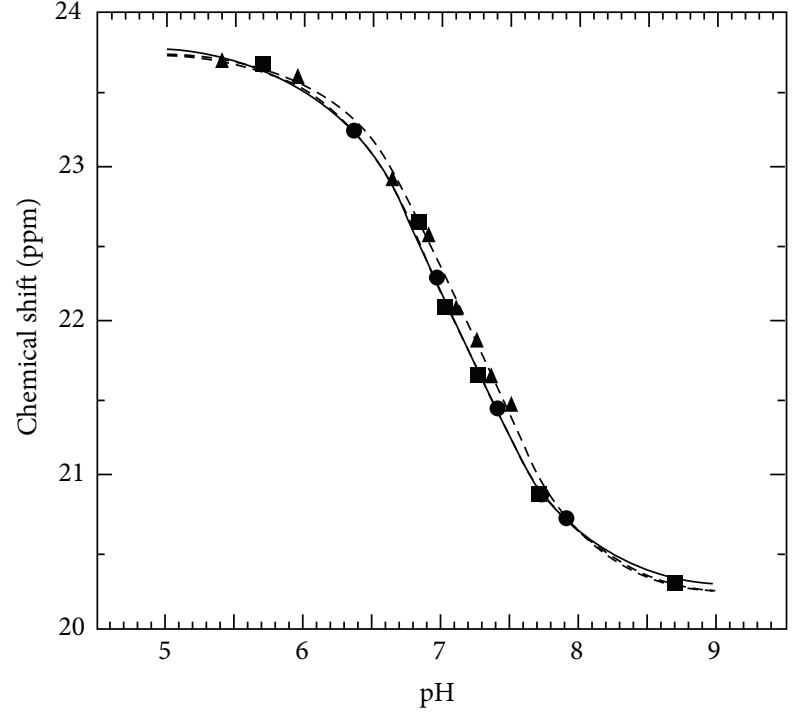

- $15^{\circ}$

- $22^{\circ}$

$\triangle 37^{\circ}$

(b)

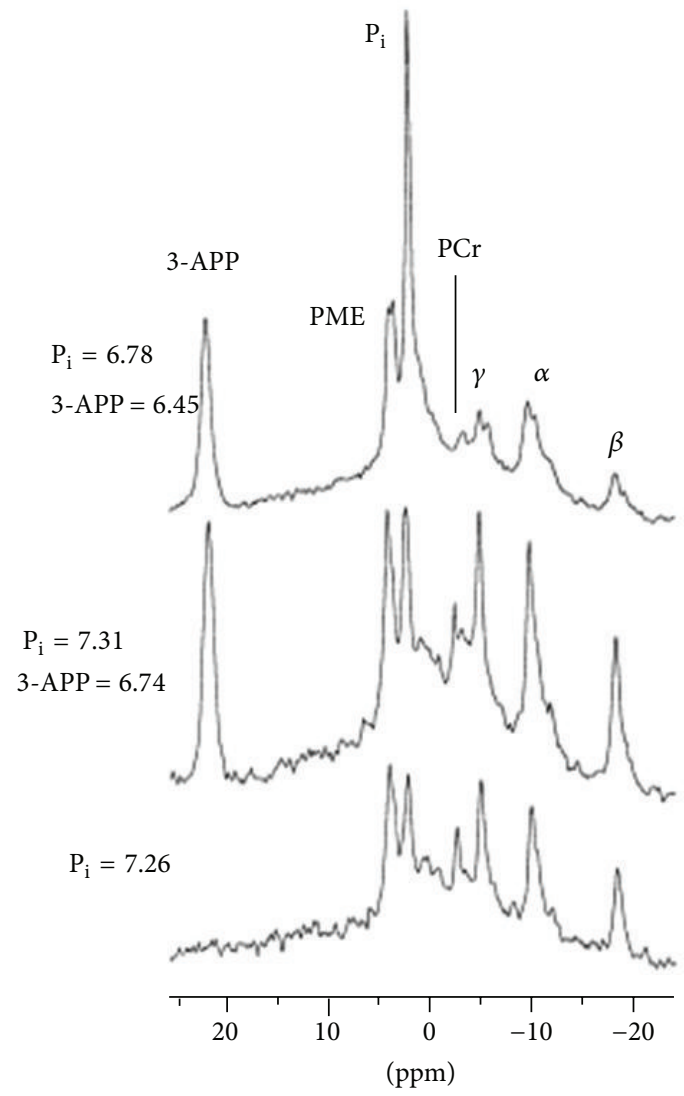

(c)

Figure 9: ${ }^{31} \mathrm{P}$ MR spectroscopy of tumor $\mathrm{pHe}$. (a) The ${ }^{31} \mathrm{P}$ spectrum from mouse leg muscle injected with 3-APP can measure extracellular $\mathrm{pH}(\mathrm{pHe})$ from the 3-APP chemical shift and can measure intracellular $\mathrm{pH}(\mathrm{pHi})$ from the chemical shift of inorganic phosphate $\left(\mathrm{P}_{\mathrm{i}}\right)$. $(\mathrm{b})$ The ${ }^{31} \mathrm{P}$ chemical shift of 3-APP is correlated with $\mathrm{pH}$ as shown by an in vitro titration. (c) The pHe and pHi measured with 3-APP and $\mathrm{P}_{\mathrm{i}}$ in a tumor before (bottom) and after (middle) injection of 3-APP, and after euthanasia (top). Reproduced with permission from [41]. 

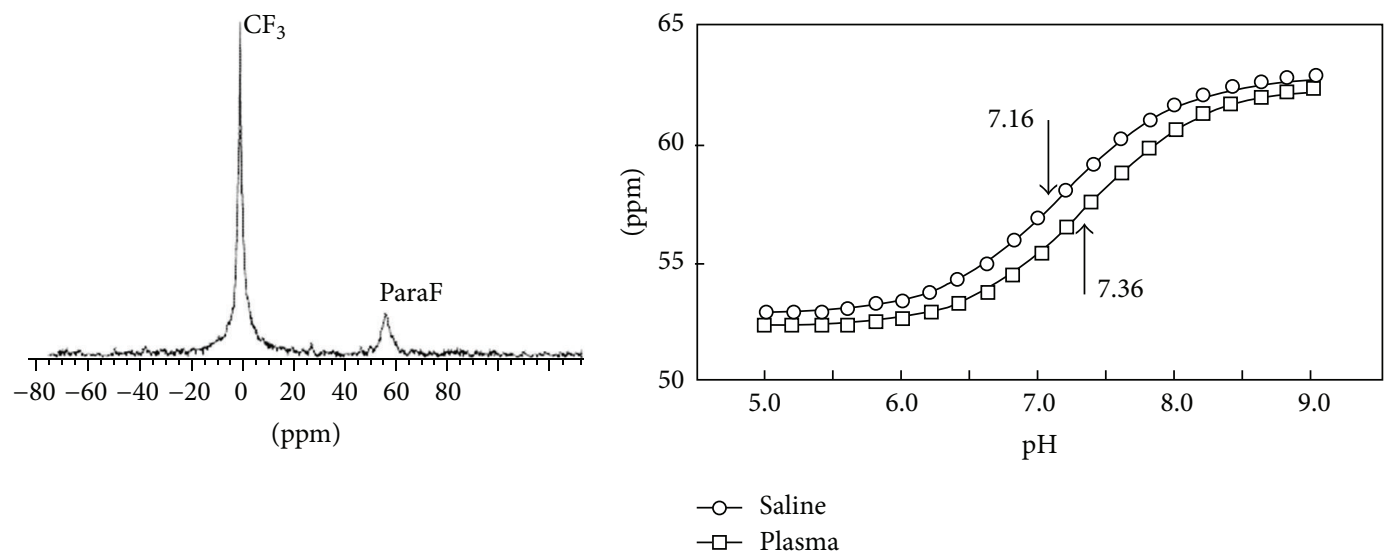

(a)

(b)

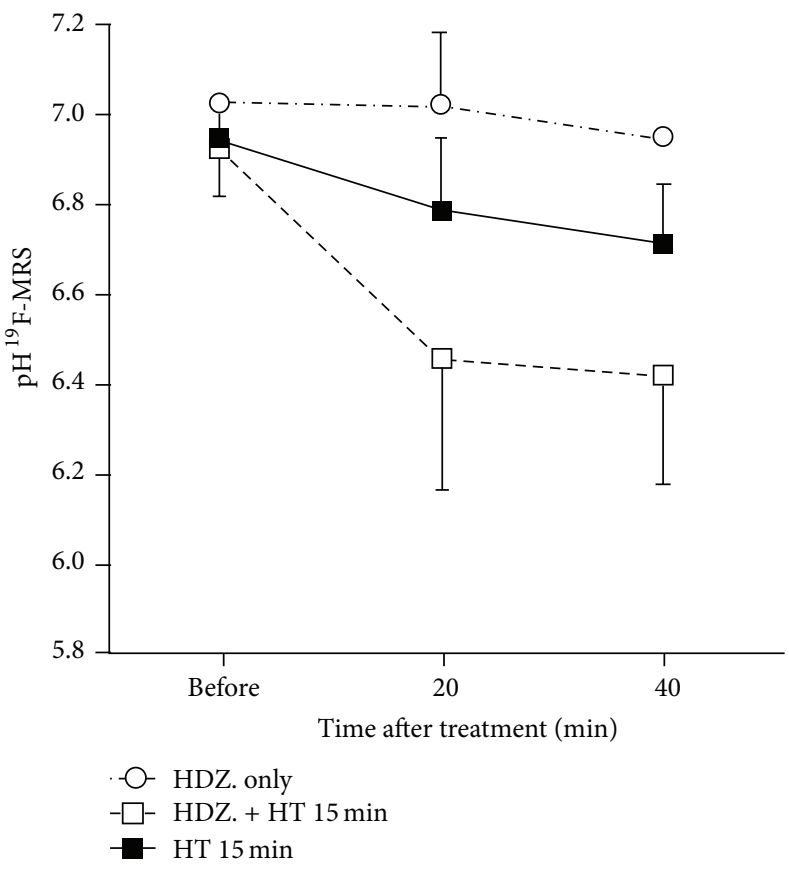

(c)

Figure 10: ${ }^{19} \mathrm{~F}$ MR spectroscopy of tumor pHe. (a) The ${ }^{19} \mathrm{~F}$ MR spectrum of ZK-150471 shows two peaks. (b) The chemical shift difference of these two peaks is correlated with $\mathrm{pH}$. (c) The average $\mathrm{pHe}$ measured with ${ }^{19} \mathrm{~F}$ MRS showed a decrease after heating with and without $5 \mathrm{mg} / \mathrm{kg}$ hydralazine. Reproduced with permission from [42].

$T_{2}$ relaxation processes of a single agent $\left[(\mathrm{GdDOTAam})_{33}-\right.$ $\mathrm{Orn}_{205}$ ] was shown to be correlated with $\mathrm{pH}$ [86]. However, this large-molecule contrast agent has not been demonstrated with in vivo studies and may not be clinically translatable because large-molecule $\mathrm{Gd}(\mathrm{III})$ chelates are potentially toxic.

\section{Chemical Exchange Saturation Transfer MRI}

A chemical exchange saturation transfer (CEST) MRI experiment can selectively detect multiple proton exchanging sites within the same contrast agent. This provides a great advantage relative to relaxivity-based MRI methods, because a ratio of CEST effects from the same CEST agent is independent of concentration. If one or both of the CEST effects are dependent on $\mathrm{pH}$, then this ratio can be used to accurately measure $\mathrm{pH}$. CEST MRI has advantages relative to other imaging methods that measure $\mathrm{pH}$. CEST MRI does not require special instrumentation such as hyperpolarizer for ${ }^{13} \mathrm{C}$ MRS or a detector coil for ${ }^{19} \mathrm{~F},{ }^{31} \mathrm{P}$, or ${ }^{13} \mathrm{C}$ MRS. CEST MRI allows full body imaging and it is not limited by depth of view as with fluorescence imaging. CEST MRI uses relatively low energy radio frequency and does not raise safety concerns like EPR or PET imaging. Most importantly, CEST MRI is noninvasive, which is a great improvement relative to invasive electrodes and microsensors. 


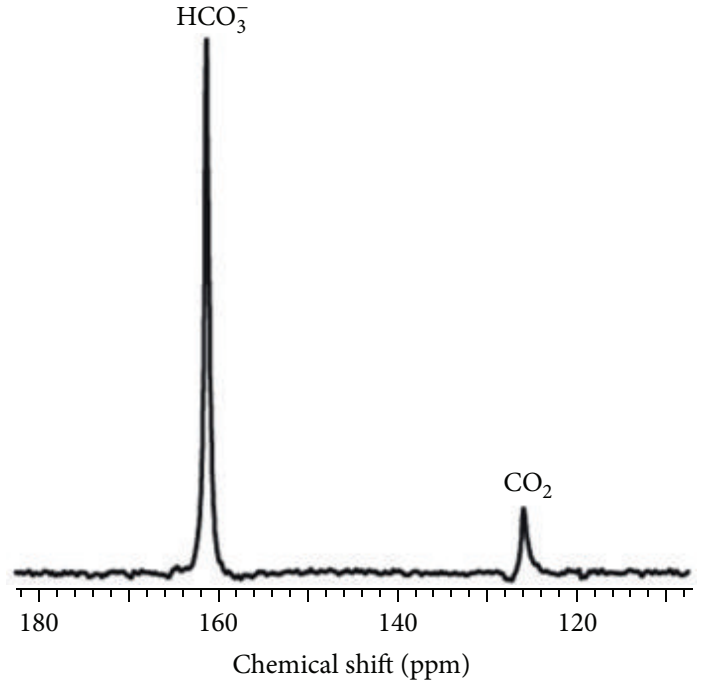

(a)

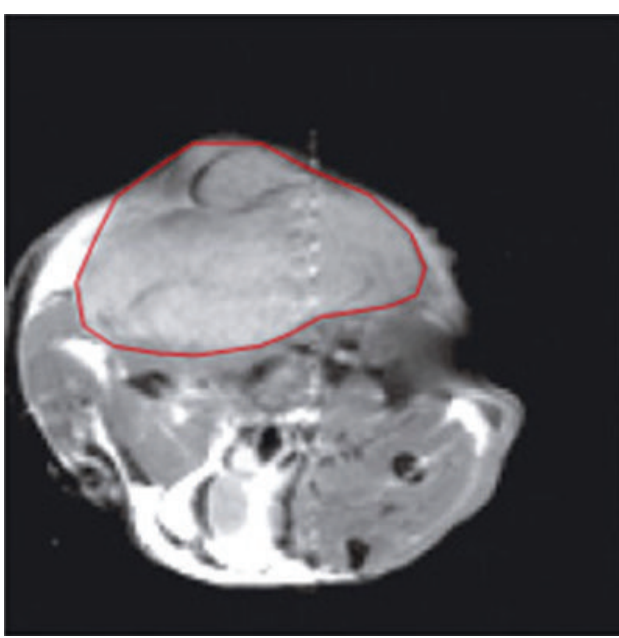

(c)

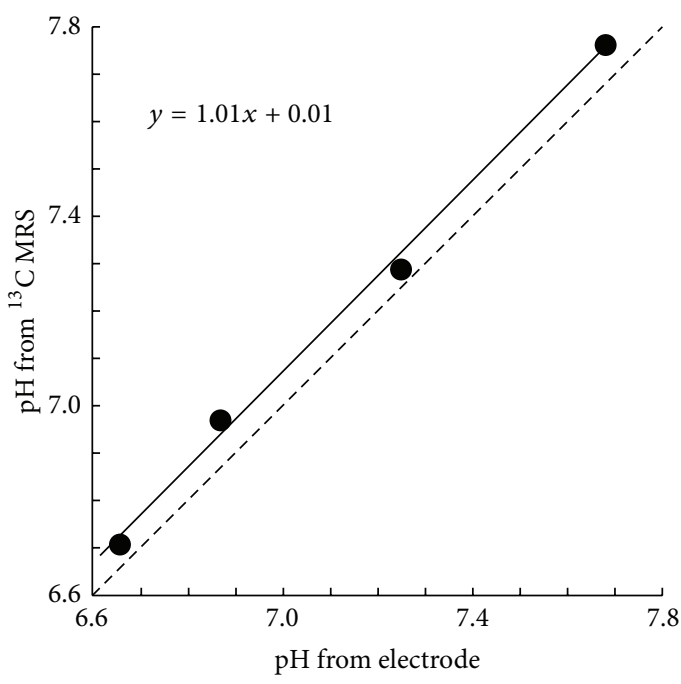

(b)

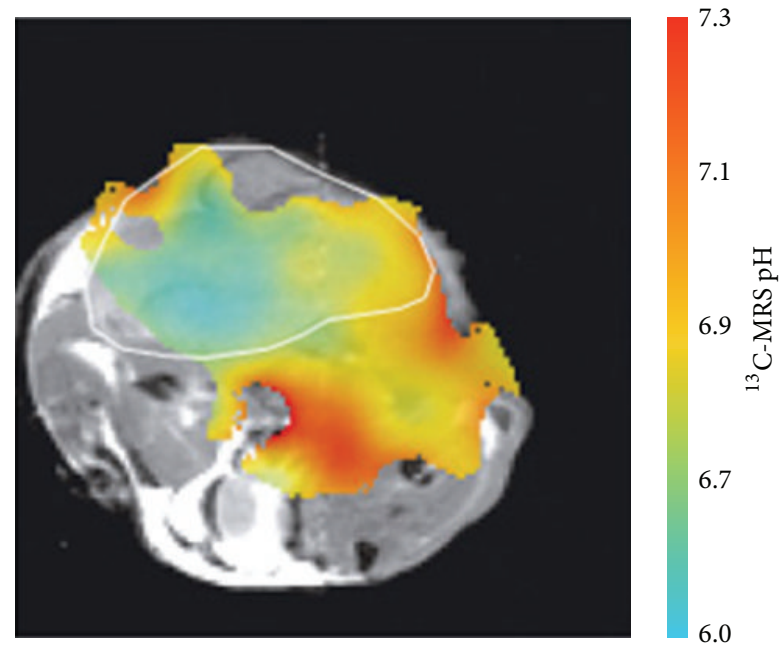

(d)

FiguRE 11: Hyperpolarized ${ }^{13} \mathrm{C}$ MR spectroscopic imaging of tumor pHe. (a) ${ }^{13} \mathrm{C}$ MR spectroscopy was used to measure the ratio of $\left[\mathrm{H}^{13} \mathrm{CO}_{3}\right] /\left[{ }^{13} \mathrm{CO}_{2}\right]$, which was used to calculate $\mathrm{pH}$ based on the Henderson-Hasselbalch equation, $\mathrm{pH}=\mathrm{pKa}+\log _{10}\left(\left[\mathrm{H}^{13} \mathrm{CO}_{3}\right] /\left[{ }^{13} \mathrm{CO}_{2}\right]\right)$. (b) A solid line shows the correlation of the $\mathrm{pH}$ values determined from ${ }^{13} \mathrm{C}$ MRS and an electrode. The dashed line representing equal measurements is provided as a visual reference. (c) A subcutaneously implanted EL4 tumor in a mouse is outlined in red in an axial MR image. (d) The $\mathrm{pH}$ map of the same mouse calculated from ${ }^{13} \mathrm{C} \mathrm{MR}$ spectroscopic imaging. The tumor margin is outlined in white. Reproduced with permission from [48].

5.1. The CEST Mechanism. CEST MRI employs an endogenous or exogenous chemical agent that has a proton which can exchange with water at a slow-to-moderate rate of $100-5000 \mathrm{~Hz}$ (Figure 13(a)). The protocol for CEST MRI starts with saturating a specific resonance frequency of an exchangeable proton on the CEST agent (Figure 13(b)). Saturation is a condition wherein the number of nuclear spin magnetic moments ("spins") aligned against the field is increased at the expense of spins aligned with an external magnetic field. This soft irradiation pulse temporary disrupts the Boltzmann distribution of spins and leads to a decrease in MRI signal amplitude of the exchangeable proton in NMR spectra, which depends on the net difference of spins aligned with and against the magnetic field [87]. Due to the natural process of chemical exchange of labile protons between molecules, this proton on the CEST agent is transferred to a nearby water molecule (Figure 13(c)). The spin aligned against the field from the exchangeable proton is transferred to the population of spins for water, in exchange for a spin aligned with the field that is transferred to the population of spins for the agent. This transfer of saturation from the exchangeable proton to the water proton reduces the MRI signal amplitude of water. In practice, the CEST MRI protocol starts with a series of selective saturation RF pulses (Figure 13(d)), followed by a standard MRI acquisition sequence that can measure the amplitude of water signal throughout the image 

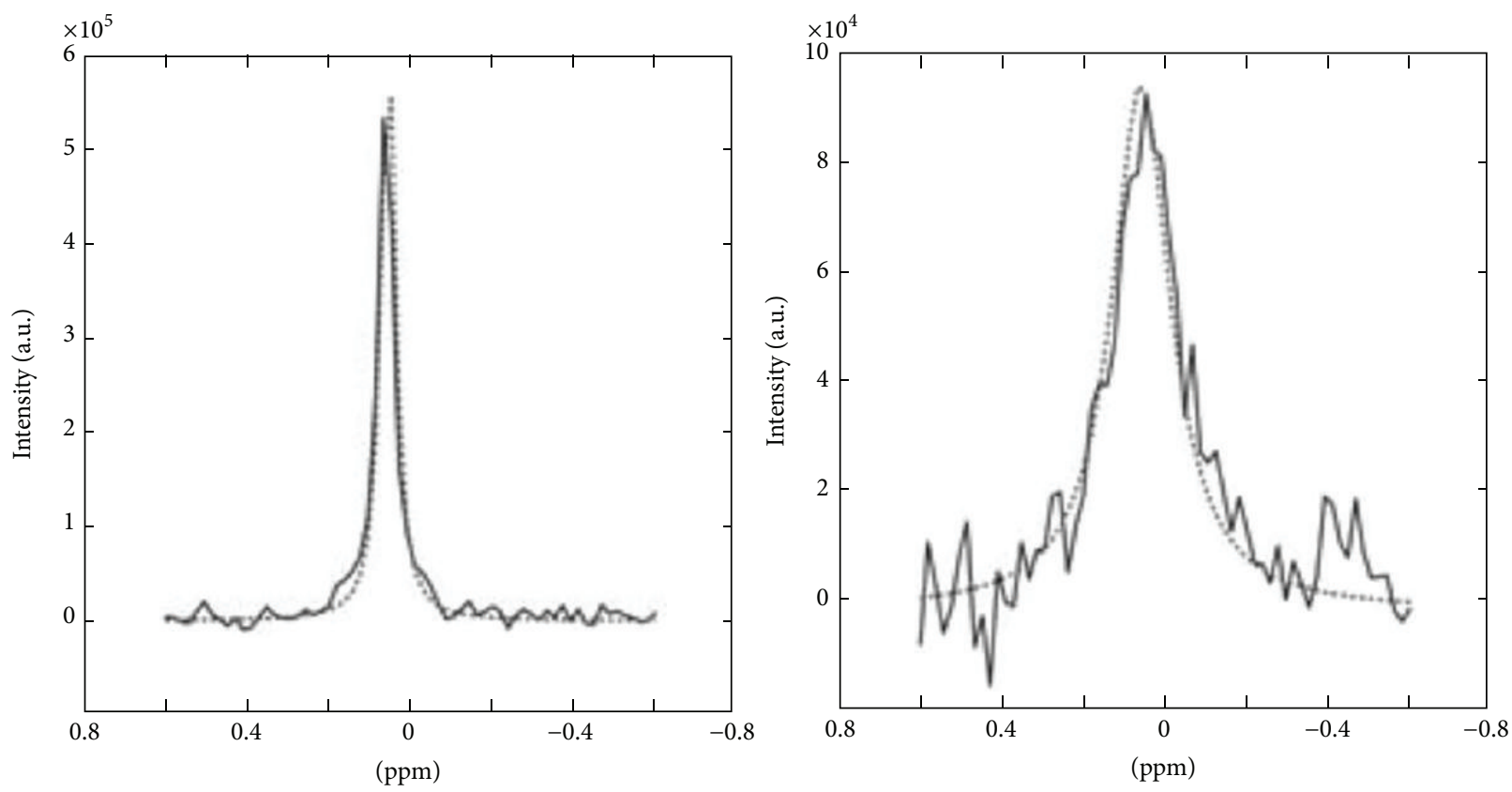

(a)
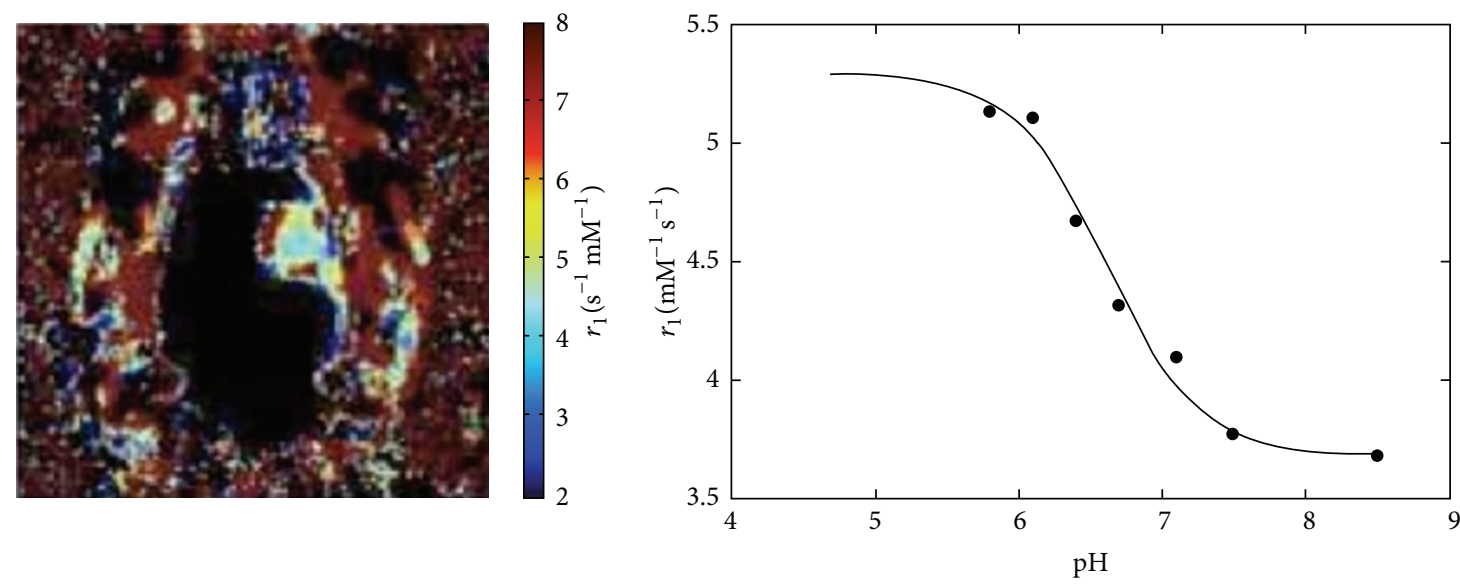

(b)

(c)

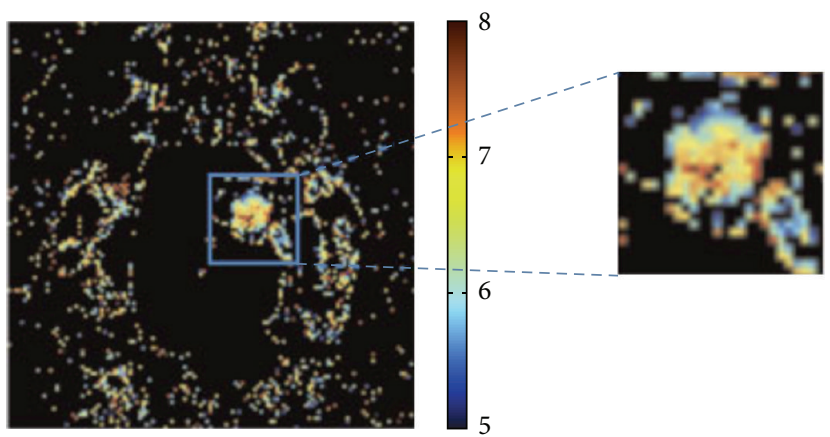

(d)

FIgURE 12: Relaxation-based MRI of Gd-DOTA-4Amp and DyDOTP can measure tumor pHe. (a) The change in water linewidth before injection (left) and after injection (right) is used to estimate the concentration of the agent. (b) A parametric map of the $r_{1}$ relaxivity of the agent in a glioma model is obtained from a $T_{1}$-weighted MR images and the concentration of the agent. (c) The $r_{1}$ relaxivity of the agent is $\mathrm{pH}$-dependent, (d) which can be used to convert the $r_{1}$ relaxivity map to a $\mathrm{pH}$ map (color scale bar shows $\mathrm{pH}$ units). Reproduced from [53] with permission. 

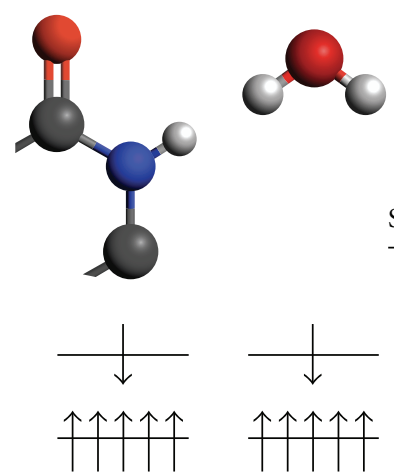

(a)

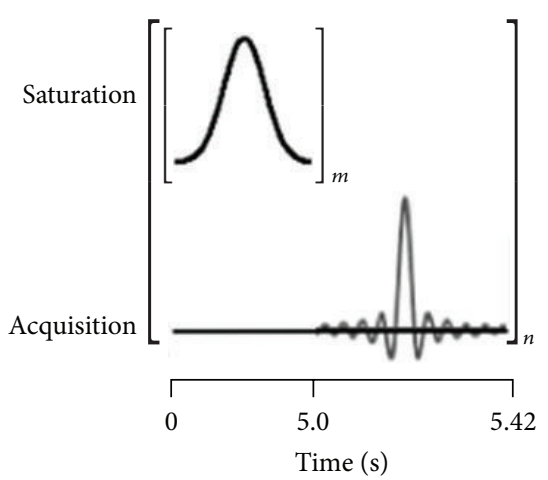

(d)

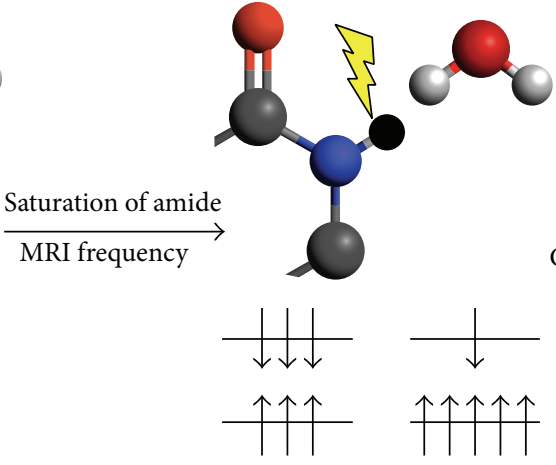

(b)

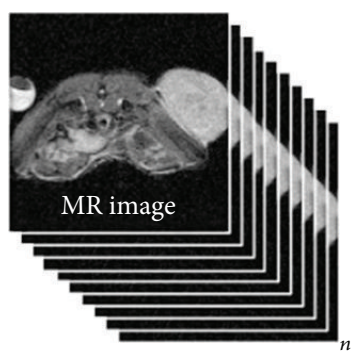

(e)

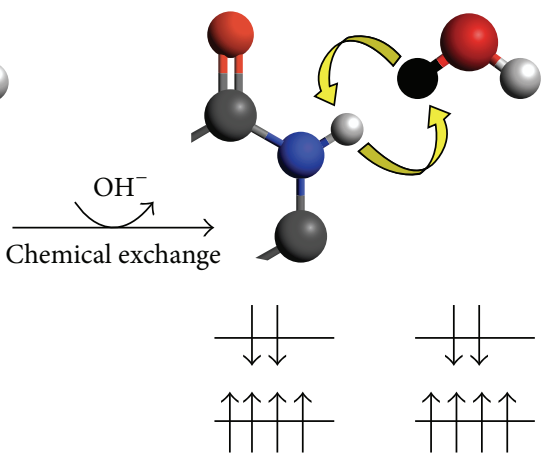

(c)

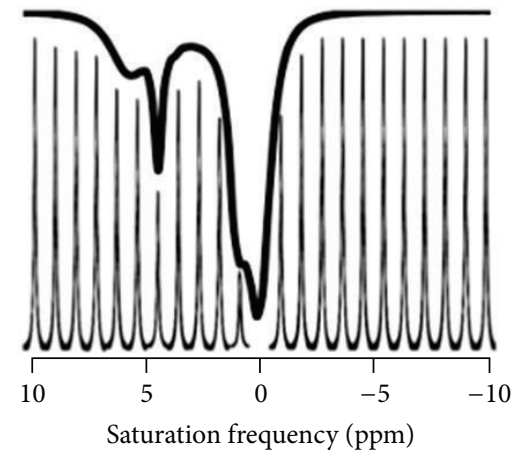

(f)

FIGURE 13: Chemical exchange saturation transfer. (a) The number of magnetic moments aligned with the B0 static magnetic field is greater than the number aligned against the B0 field for an amide proton and a water proton. (b) Selective saturation of the MR frequency of the amide proton causes the magnetic moments to equilibrate between states. (c) Subsequent chemical exchange of the amide proton and water proton transfers some of the saturation to the water protons, causing a partial equilibration of the states for the water protons. (d) The CEST-FISP MRI protocol consists of a series of Gaussian-shaped saturation pulses repeated " $m$ " times, followed by a FISP MRI acquisition sequence. The entire process is repeated for a series of " $n$ " saturation frequencies. (e) Fourier transformation of the frequency-domain signals creates a series of " $n$ " MR images at each saturation frequency. (f) The integral of the MR signal of the tumor is plotted as a function of saturation frequency for the " $n$ " images, creating a CEST spectrum. A sum of four Lorentzian line shapes was fit to the experimental CEST spectrum to quantify the CEST effects at 5.6, 4.2, and $0.8 \mathrm{ppm}$ of the CEST agent used in this example (iopromide; Ultravist, Bayer Healthcare Inc.) and also account for the direct saturation of water at $0 \mathrm{ppm}$ (black line, raised 10\% above the CEST spectral peaks to improve the view).

(Figures 13(d) and 13(e)). A CEST spectrum is obtained by iterating a series of saturation frequencies and recoding the normalized water signal amplitudes (Figures 13(e) and 13(f)).

The CEST agent typically has a low concentration in the 1 to $10 \mathrm{mM}$ range. The MR spectroscopic resonances of the agent are not directly observable during a standard MRS acquisition due to the low concentration of agent relative to the concentration of water. However, with continuous RF irradiation (Figure 13(d)), the saturation from the agent is continuously transferred to the bulk water (Figure 13(c)), resulting in a reduction of water intensity that is observable by MRI (Figures 13(e) and 13(f)). Hence, this continuous transfer of saturation serves as amplification for the agent and allows for indirect observation of the CEST agent at low $\mathrm{mM}$ concentrations [88].

5.2. Endogenous CEST MRI. An ingenious application of CEST is to detect exchanging groups that are already present in the tissue, such as hydroxyl, amide and amine groups in proteins and peptides. Endogenous CEST avoids the need of an exogenous contrast agent, resulting in high impact in both in vivo applications and clinical translatability. For example, glycoCEST detects hydroxyls $(0.5-1.5 \mathrm{ppm})$ from glycogen in liver and muscle [89], gagCEST detects hydroxyls (0.9$1.5 \mathrm{ppm}$ ) from glycosaminoglycan in cartilage [90], gluCEST detects amines (3.0 ppm) from glutamate in brain [91], and amide proton transfer (APT) detects amides (3.5 ppm) from proteins and peptides in brain [59].

Among the established endogenous CEST methods studied, only APT can measure differences in $\mathrm{pH}$. The chemical exchange between the amide protons and the bulk water is base-catalyzed, so that the exchange rate decreases with decreasing $\mathrm{pH}$. The $\mathrm{pH}$-weighted APT imaging method has been widely used to study acute ischemic stroke in an animal model (Figure 14) [64, 83, 84, 92-94].

When using APT to evaluate $\mathrm{pH}$, the generated contrast depends on many parameters which compromises the measurement of $\mathrm{pH}$. These other parameters include water 


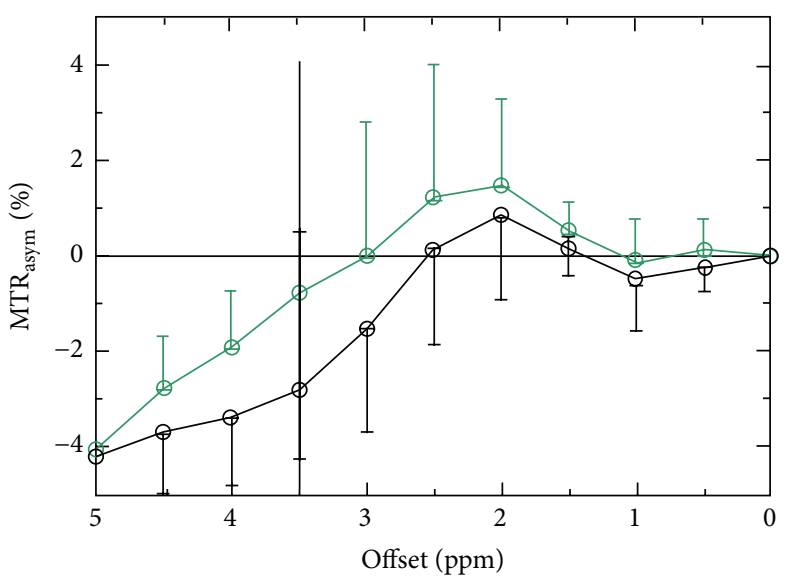

(a)

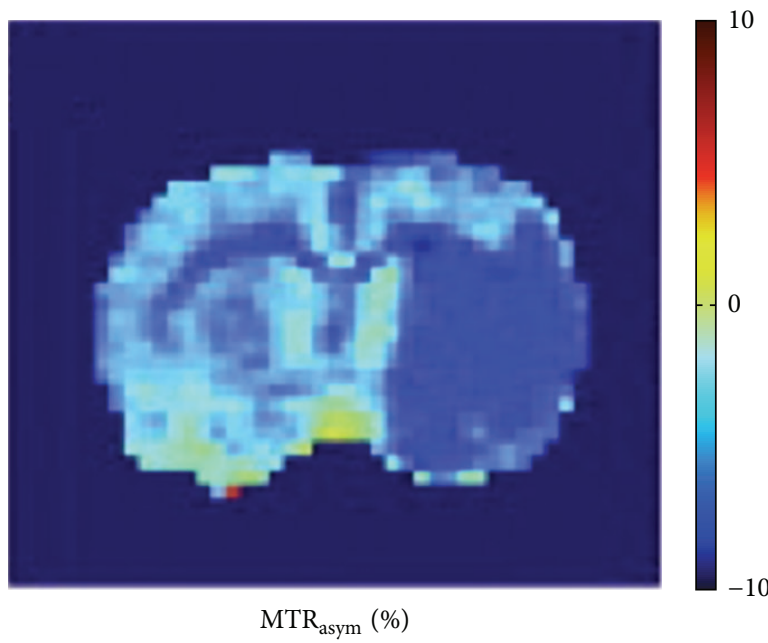

(c)

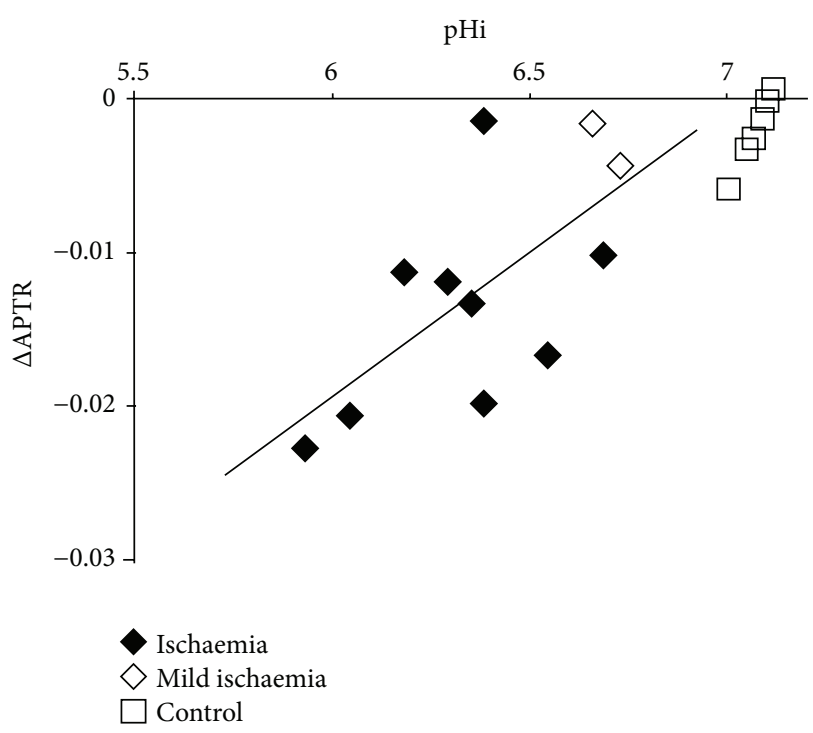

(b)

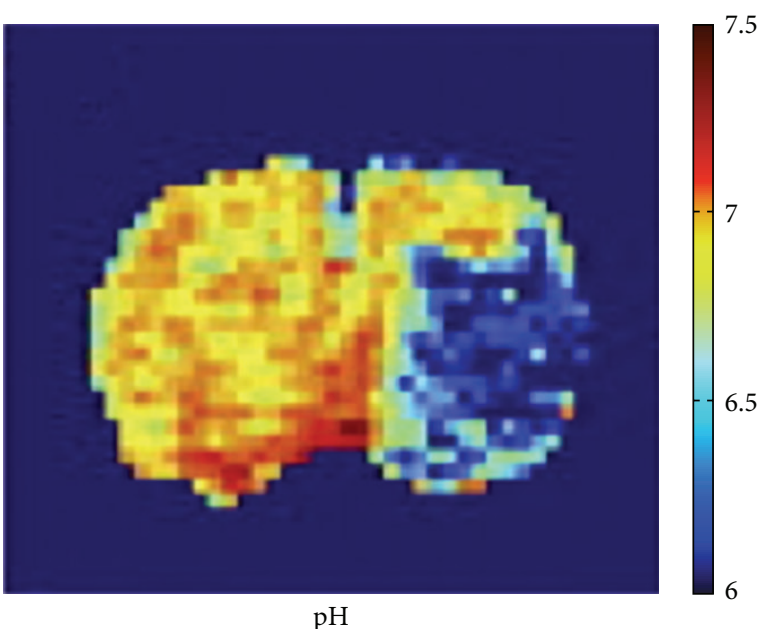

(d)

FIGURE 14: Endogenous CEST MRI of tumor pHi. (a) The asymmetry of the magnetization transfer ratio (MTR asym $)$, a measure of CEST, decreases after ischemia is induced in a rat model (black: ischemic region; green: nonischemic contralateral region; data represents the average and standard deviation of results with 7 rats). (b) This decrease in CEST, also represented as a change in the amide proton transfer ration ( $\triangle$ APTR), has been correlated with intracellular $\mathrm{pH}\left(\mathrm{pHi}\right.$ ) as measured with ${ }^{31} \mathrm{P} \mathrm{MR}$ spectroscopy. (c) The MTR asym can be mapped in a rat model of ischemia, (d) which can be converted into a $\mathrm{pH}$ map. Reproduced with permission from $[59,83,84]$.

proton concentration, amide proton concentration, spinlattice relaxation rate, and saturation time when measuring CEST via $\mathrm{MTR}_{\text {asym }}$ at $3.5 \mathrm{ppm}$ (Equation (1)) [59]. For this reason, the $\mathrm{pH}$-weighted APT image can measure relative changes in $\mathrm{pH}$, but no absolute $\mathrm{pH}$ value can be calculated. In the past, $\mathrm{pH}$-weighted APT images have been correlated with apparent diffusion coefficient (ADC) images [64, 84, 94], isotropic diffusion-weighted images $[59,95]$, and lactic acid content using nonimaging methods $[83,94]$. Alternative quantification methods have been applied, such as calibrating with various $\mathrm{pH}$ solutions of creatine [60], cross-reference with ${ }^{31} \mathrm{P}$ MRS [59], and employing QUEST/QUESP to establish a relationship between the amide proton transfer ratio (APTR) and $\mathrm{pH}$ [96]. Consider

$$
\begin{aligned}
\operatorname{APTR}(3.5 \mathrm{ppm})= & \operatorname{MTR}_{\text {asym }}(3.5 \mathrm{ppm}) \\
& +\frac{[\text { amide proton }]}{[\text { water proton }]} \cdot \frac{\left(1-e^{-R_{1 w} t_{\text {sat }}}\right)}{R_{1 w}} \\
& \cdot k_{\text {base }} \cdot 10^{\mathrm{pH}-\mathrm{pK}_{w}} .
\end{aligned}
$$




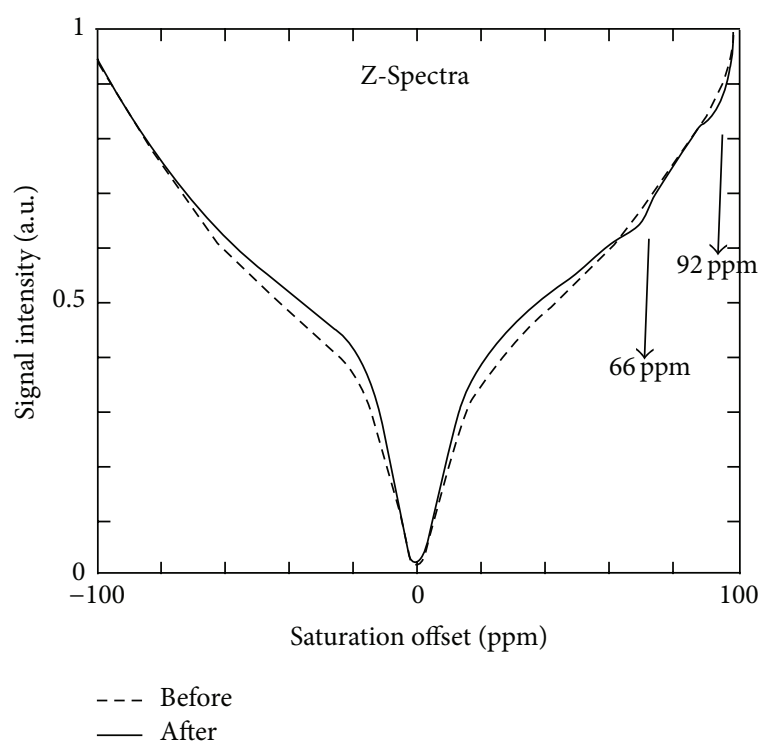

(a)

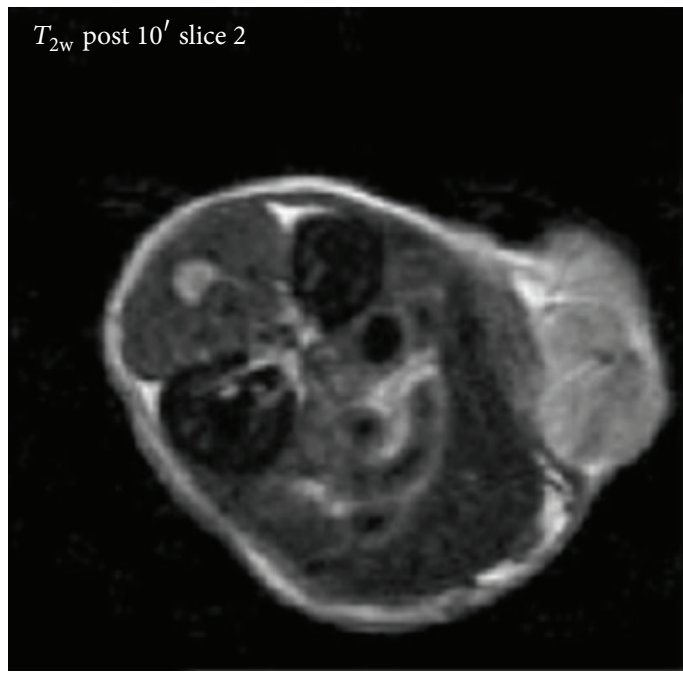

(c)

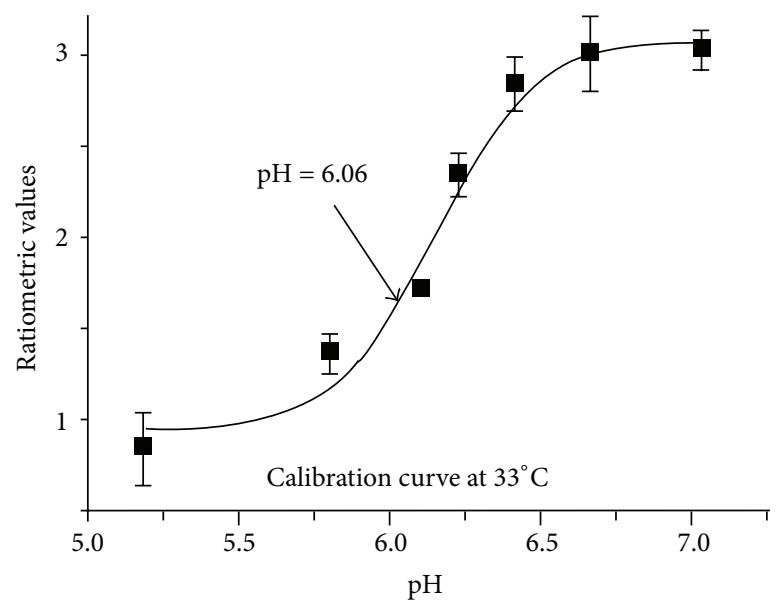

(b)

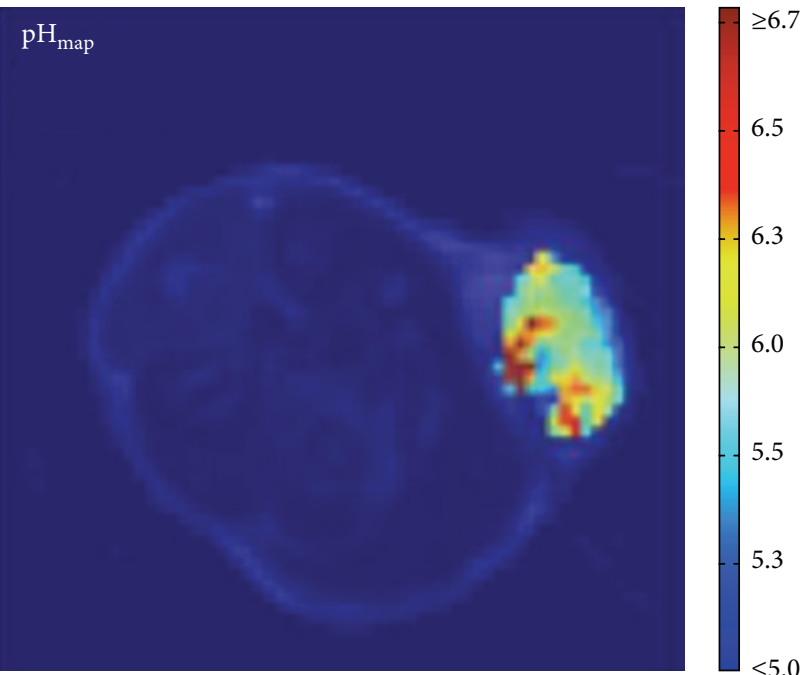

(d)

FIGURE 15: CEST MRI of tumor pHe with Yb-HPDO3A. (a) An in vivo CEST spectrum of the tumor mass before and after i.v. injection of the agent into a mouse model of B16-F10 melanoma. (b) A ratio of the two CEST effects is calibrated with $\mathrm{pH}$ at $33^{\circ} \mathrm{C}$. Although the calibration is dependent on temperature, the temperature can be determined from the chemical shifts of the CEST effects. (c) An anatomical image shows the location of the subcutaneous tumor. (d) The pixelwise pHe map of the tumor shows a heterogenous distribution of pHe values with an average $\mathrm{pHe}$ of 5.8. Reproduced with permission from [61].

Due to the high concentration of mobile proteins and peptides in the cytoplasm, APT mainly measures intracellular $\mathrm{pH}$ [59]. Furthermore, as shown in (1), an increase in APTR can potentially be caused by an increase in cytosolic protein and peptide content rather than intracellular $\mathrm{pH}$. In fact, the increased protein and peptide content in the tumor is the most likely explanation for changes in APT contrast in tumors, because the intracellular $\mathrm{pH}$ is highly regulated by active proton exporting systems [5] and there is usually only a small difference $(<0.1 \mathrm{pH}$ unit) between a malignant tumor and normal tissue. Thus, APT imaging would be a more appropriate tool to provide visual information about the presence and grade of tumor based on increased content of mobile proteins and peptides, as shown in past clinical studies $[60,97]$.

5.3. Exogenous PARACEST Agents. Paramagnetic Gd(III) is the most commonly used contrast agent for MRI because it is the most efficient at relaxing bulk water protons, providing excellent contrast in a $T_{1}$ weighted image. However, having such a rapid water relaxation does not allow for sufficient time to generate CEST between an agent and water, which 


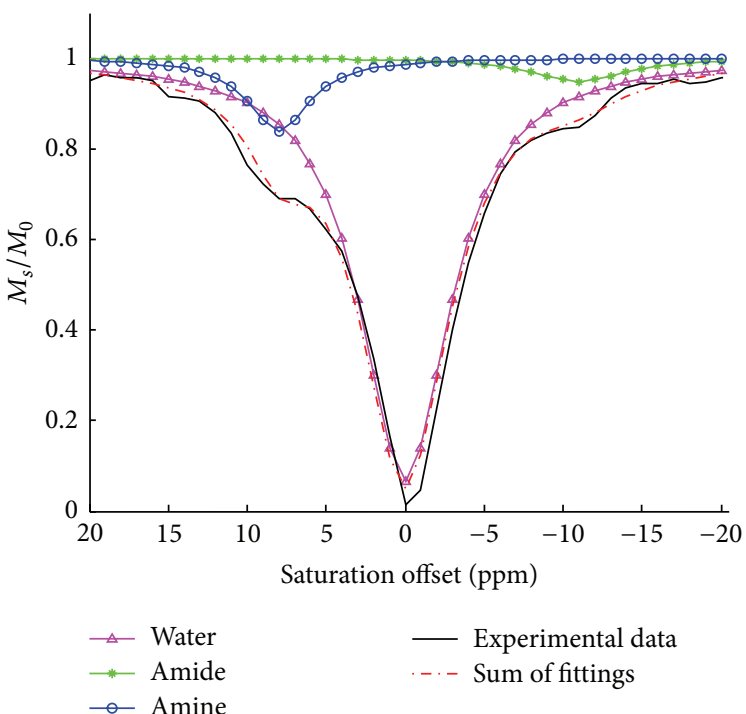

(a)

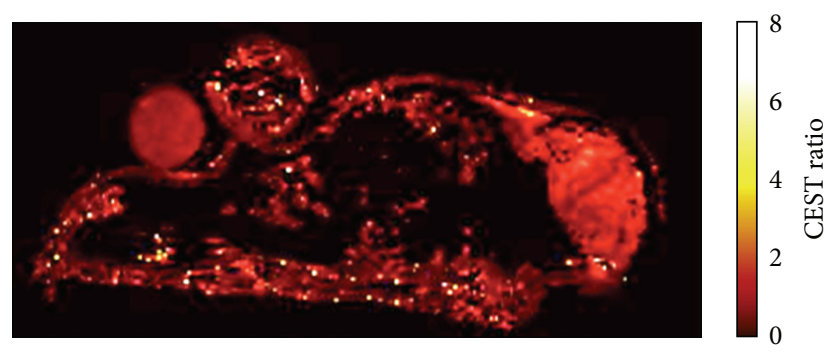

(c)

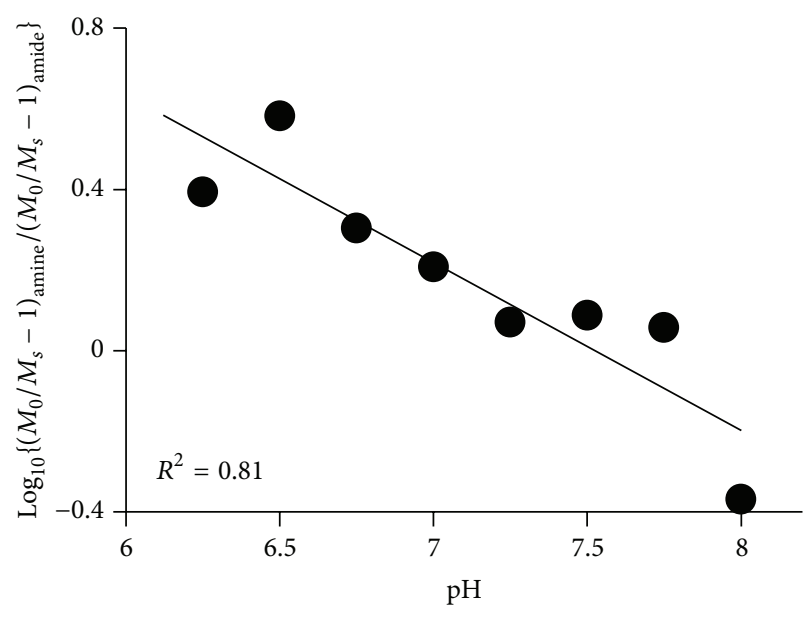

(b)

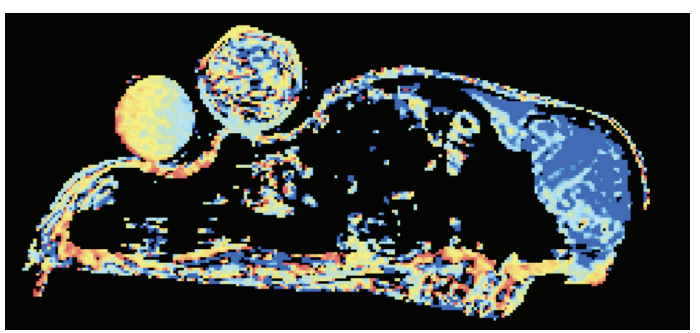

(d)

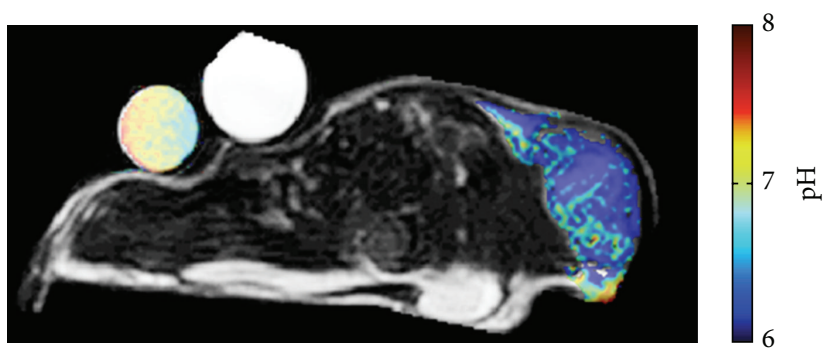

(e)

FIGURE 16: CEST MRI of tumor pHe with Yb-DO3A-oAA. (a) The CEST spectrum of Yb-DO3A-oAA was fitted with Lorentzian line shapes to measure two CEST effects. (b) A ratio of the CEST effects was linearly correlated with pH. (c) The same CEST ratio was measured in a MCF-7 mammary carcinoma model after direct injection of the agent into the tumor tissue. (d) A map of extracellular $\mathrm{pH}$ ( $\mathrm{pHe}$ ) was determined from the map of the CEST ratio. (e) The pHe map was filtered to only retain results from pixels that had two statistically significant CEST amplitudes, resulting in a pHe map of the tumor and tube containing the agent (the other tube contained only water). Reproduced with permission from [57].

makes it unsuitable for CEST detection. On the contrary, other lanthanide ions have slower exchange rates and are more suitable as PARACEST agents [99].

PARACEST agents contain a lanthanide ion that greatly shifts the MR resonance frequency of the exchangeable amide protons from the MR frequency of water, which expands the range of MR frequencies that can generate a CEST effect, away from the direct saturation of water experienced in the
APT experiment. This expanded frequency range facilitates the development of an agent with two CEST effects that have different MR frequencies. Due to the variety of the chelation chemistry, PARACEST agents can be designed to possess both $\mathrm{pH}$-responsive and $\mathrm{pH}$-unresponsive CEST effects within a single agent. A ratio of the two CEST effects can then be used to measure pHe without complications of the concentration term (Equation (2)) [100], unlike 


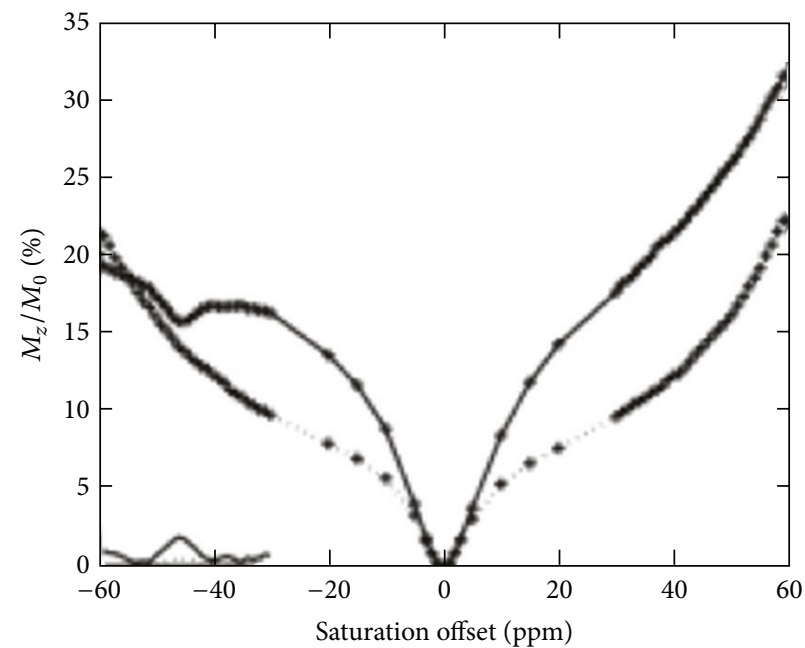

+ Preinjection

$\rightarrow$ Postinjection

(a)

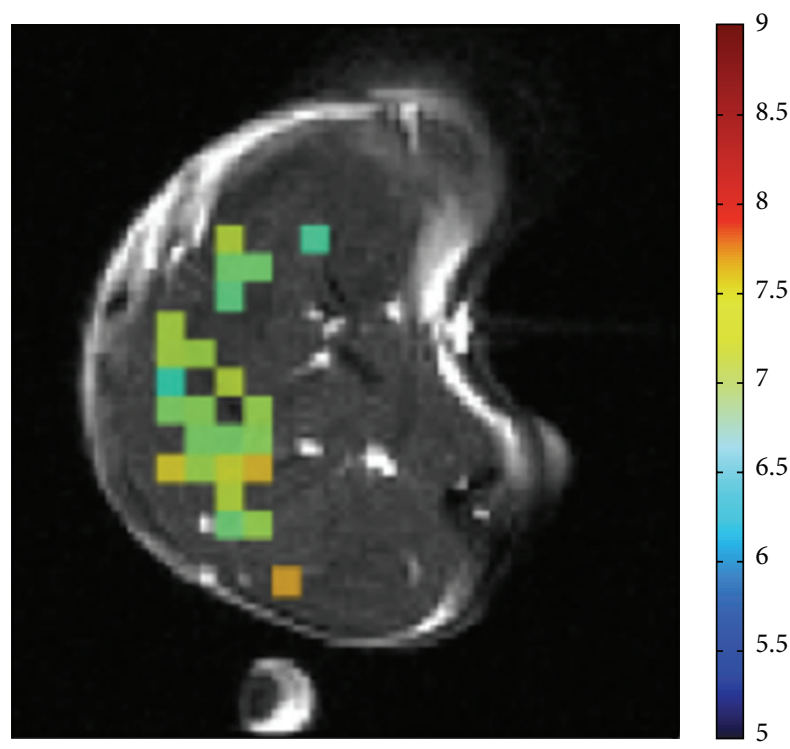

(c)

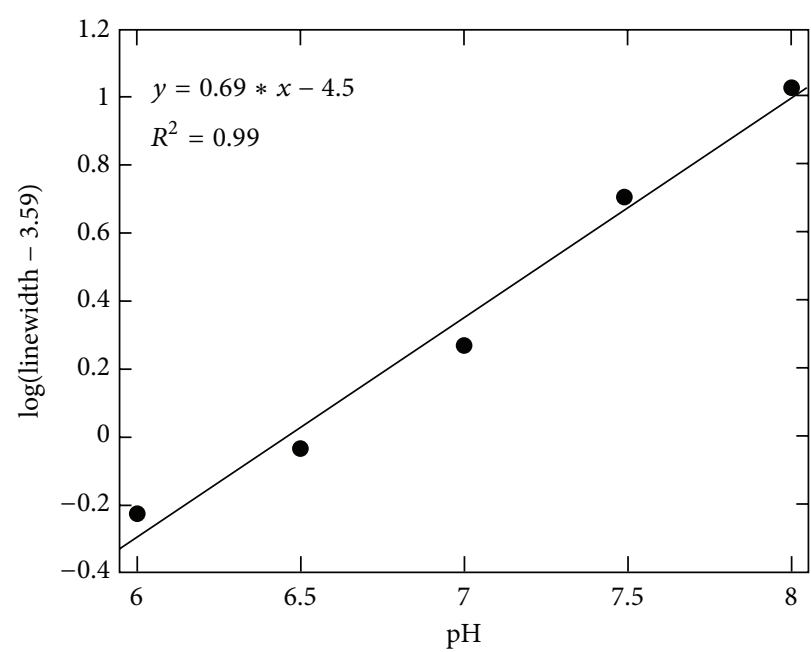

- Experimental

— Linear fit

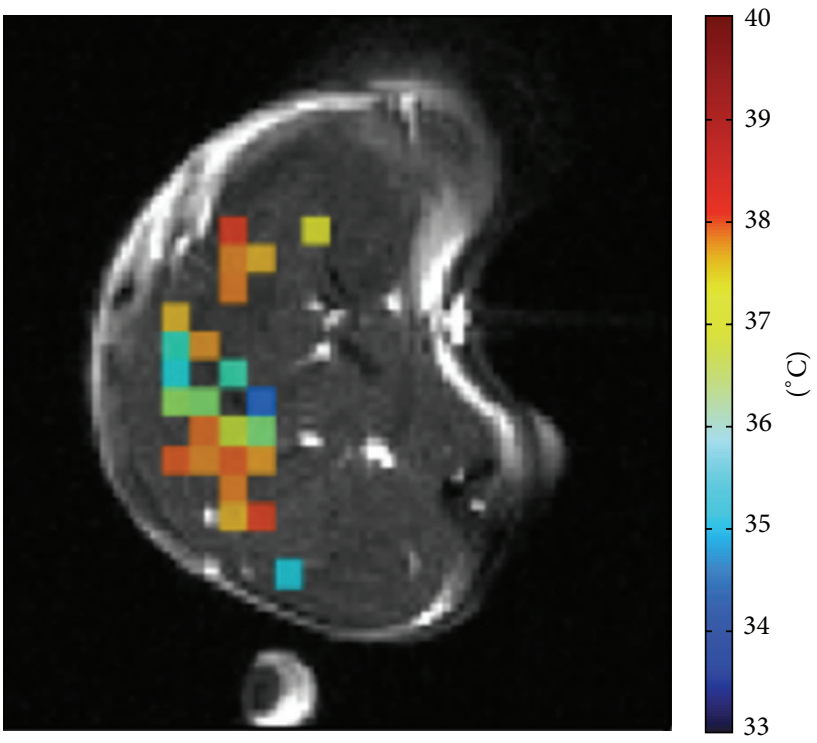

(d)

FIGURE 17: CEST MRI of leg pHe with Tm-DOTAM-Gly-Lys. (a) An in vivo CEST spectrum of the tumor mass before and after direct injection of the agent into the left mouse leg. (b) The linewidth of the CEST effect is calibrated with $\mathrm{pH}$. (c) The in vivo pHe map and (d) the in vivo temperature map are superimposed onto a preinjection anatomical image. The temperature is determined from the chemical shift of the CEST effect. Reproduced with permission from $[62,63]$.

approaches with Gd(III) pH-responsive relaxivity agent or APT. Consider

$$
\frac{\left[\left(M_{0}-M_{s}\right) / M_{s}\right]_{\mathrm{CA} 1}}{\left[\left(M_{0}-M_{s}\right) / M_{s}\right]_{\mathrm{CA} 2}}=\frac{n_{\mathrm{CA} 2} k_{\mathrm{CA} 2}}{n_{\mathrm{CA} 1} k_{\mathrm{CA} 1}} .
$$

Aime and colleagues were the first to design a series of pH-responsive ratiometric PARACEST agents (Ln-DOTAMGly, Ln = Pr, Nd, Eu) [54-56]. The CEST effect from an amide is $\mathrm{pH}$-responsive while CEST from the metal-bound water is $\mathrm{pH}$-unresponsive. The ratio of CEST effects from Pr-DOTAM-Gly gave the most sensitive $\mathrm{pH}$ response over a range of $\mathrm{pH}$ 5.5-7.5. Unfortunately, owing to fast exchange rate of metal-bound water at physiologic temperature, high saturation of $87.6 \mu \mathrm{T}$ power was required [56]. Such high saturation power exceeds the specific absorption rate (SAR) and limits the agent's applicability for in vivo tumor $\mathrm{pHe}$ measurements.

To avoid detecting the CEST effect from a metal-bound water, the same group measured the CEST effects of hydroxyl groups in $\mathrm{Yb}-\mathrm{HPDO} 3 \mathrm{~A}$, an analogue to $\mathrm{FDA}$ approved ProHance (Gd-HPDO3A) (Figure 15) [61, 101]. The ratio of hydroxyl CEST effects arises from the two isomeric forms 


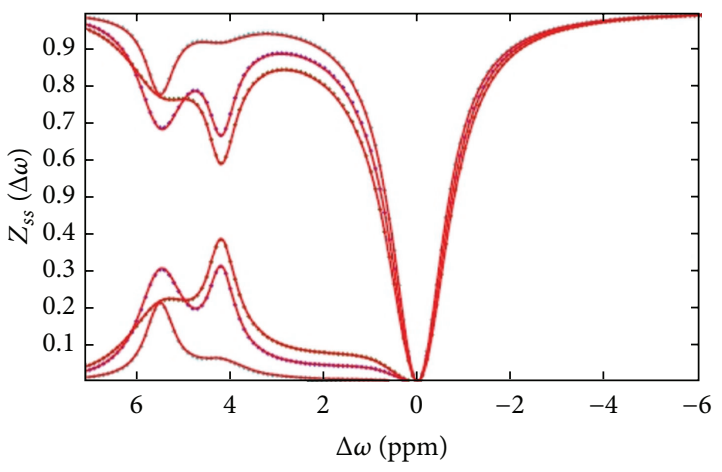

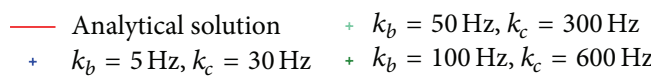

(a)

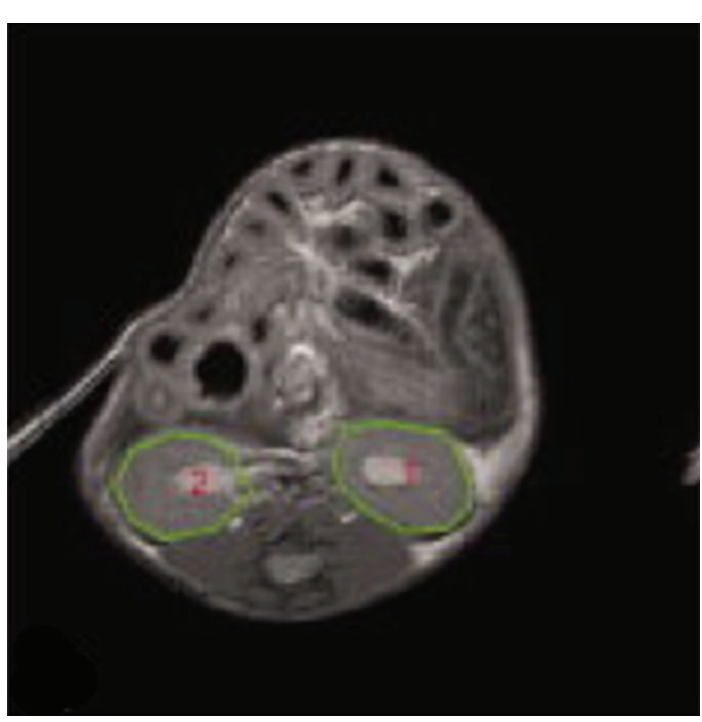

(c)

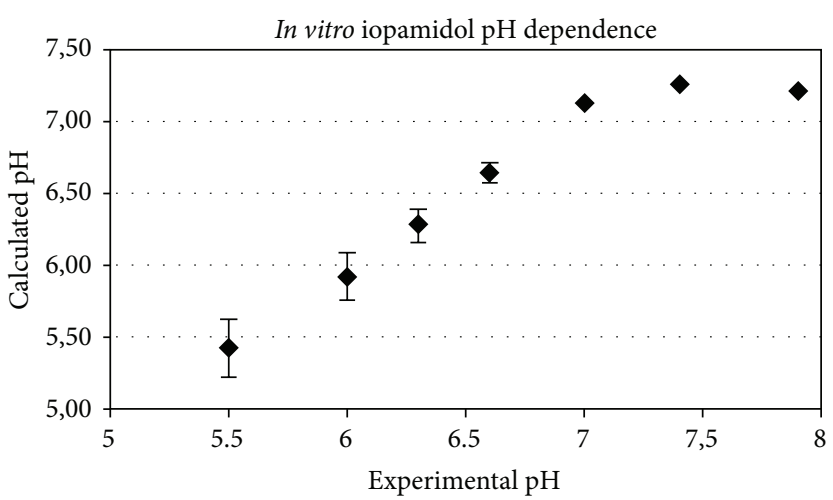

(b)

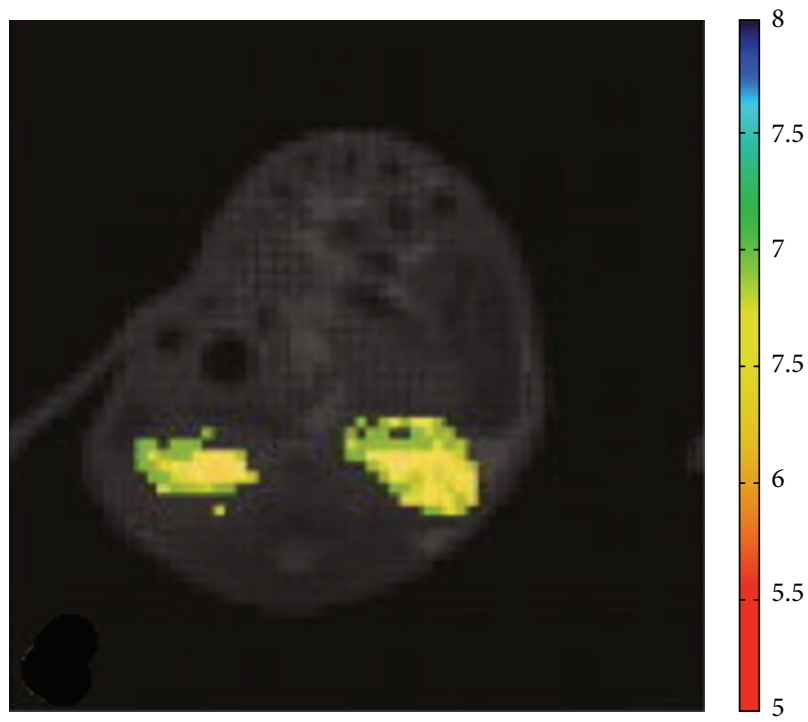

(d)

FIGURE 18: CEST MRI of tumor pHe with iopamidol. (a) A CEST spectrum the agent shows two CEST effects at 4.2 and 5.5 ppm. Both CEST effects are dependent on saturation conditions, which indicates that CEST MRI measurements of $\mathrm{pH}$ should be performed with optimized saturation conditions for best results. (b) The calculated $\mathrm{pH}$ based on a ratio of the CEST effects is correlated with experimental $\mathrm{pH}$ measured with an electrode. (c) An anatomical image shows the locations of the kidneys. (d) The pixelwise pHe map of the kidneys shows a homogenous distribution of $\mathrm{pHe}$ values with an average $\mathrm{pHe}$ of 5.8. Reproduced with permission from $[66,98]$.

and was linearly correlated within the $\mathrm{pH}$ range of 5.2-6.7. Using this agent, the pHe of early and late stage murine melanoma flank tumors were measured to be $\mathrm{pH} 6.1$ and 5.8 , respectively. Although this approach is promising, these pHe values are far below the typical range of acidic tumors, which ranges from $\mathrm{pH} 6.4$ to 7.2 . Therefore, this result may have a systematic error that produces $\mathrm{pH}$ measurements that are too low. Another limitation is that Yb-HPDO3A can only measure pHe below $\mathrm{pH}$ 6.7, which is less than the physiologic $\mathrm{pH}$ range for tumors and normal tissues. An interesting variation of $\mathrm{pH}$ measurements with $\mathrm{Yb}-\mathrm{HPDO} 3 \mathrm{~A}$ used a PAMAM dendrimer to carry multiple Yb-HPDO3A monomers [57].

Our research group has previously developed $\mathrm{Yb}-\mathrm{DO} 3 \mathrm{~A}-$ oAA that has an aryl amine and amide (Figure 16) $[65,100]$. The chemical exchange rate of an aryl amine is sufficiently slower due to the hydrogen bonding to proximal carboxylates. This allows the use of lower saturation powers $(20 \mu \mathrm{T}$ [102]) that are safer for in vivo preclinical measurements of tumor pHe. In addition, the base-catalyzed chemical exchange rates of an amide and aryl amine are different, and a ratio of these CEST effects is linearly correlated with $\mathrm{pH}$ within the range of 6.35-7.57. Using this agent, the pHe measured in a mammary carcinoma flank tumor was $\mathrm{pH} 6.8 \pm 0.21$, which is well within the physiologic $\mathrm{pH}$ range [65]. Nonetheless, the use of the lanthanide ion and $20 \mu \mathrm{T}$ saturation power is not considered safe for clinical translatability.

An alternative to measuring the magnitude of two CEST peaks is measuring a ratio of CEST at two frequencies. Wu and colleagues took a ratio of CEST signals at 55 and $49 \mathrm{ppm}$ of Eu-DO3A-tris(amide), which only has one CEST effect 


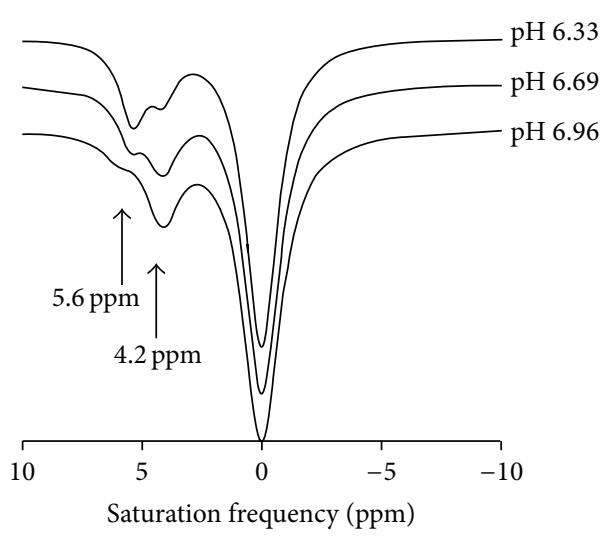

(a)

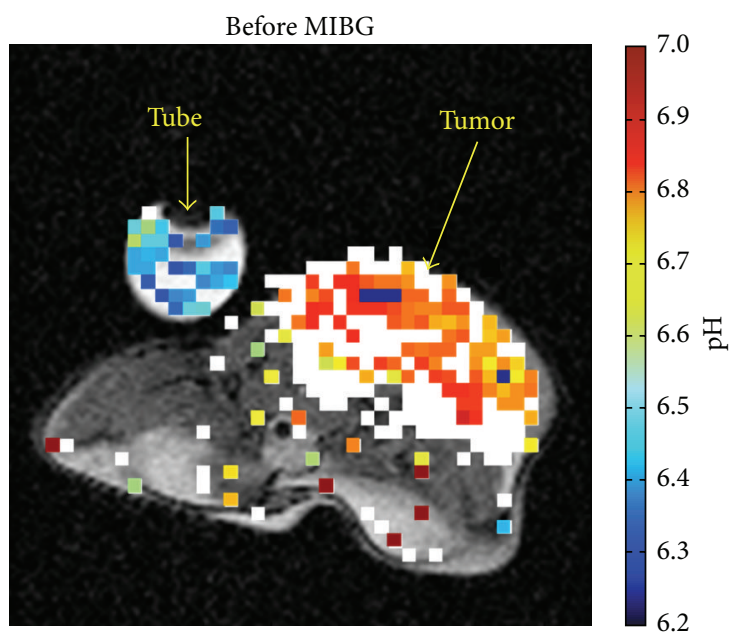

(c)

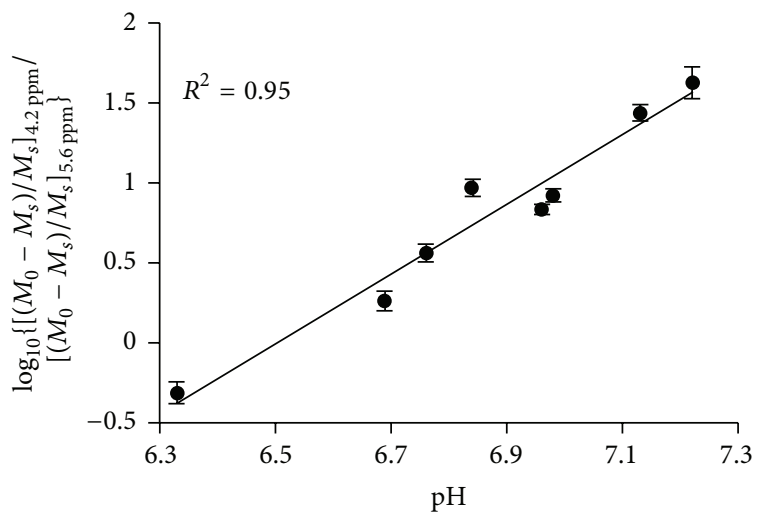

(b)

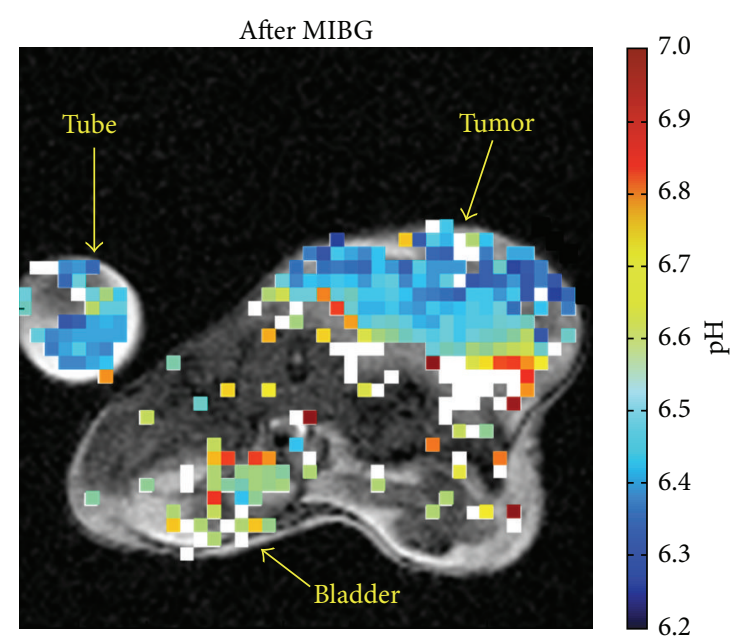

(d)

FIGURE 19: Tumor pHe measured with acidoCEST MRI using iopromide. (a) The CEST spectra of the agent show that the two CEST effects at 4.2 and $5.6 \mathrm{ppm}$ are dependent on $\mathrm{pH}$. (b) $\mathrm{A} \log _{10}$ ratio of the two CEST effects is linearly correlated with $\mathrm{pH}$ as measured with a microelectrode. (c, d) The pixelwise pHe map of a mouse bearing a Raji xenograft tumor before and after treatment with MIBG, a midrocondrial poison that causes acidification. Colored pixels have acidic pHe values $\leq 7.0$ that correspond to the color-bar. White pixels represent tumor regions with only a single CEST effect at $4.2 \mathrm{ppm}$, which were considered to have neutral pHe values $>7.0$. Reproduced with permission from [68].

that varies with $\mathrm{pH}$ between these ppm values [103]. A ratio of CEST at 55 and $49 \mathrm{ppm}$ is linearly correlated with $\mathrm{pH}$ within the range of 6.0 and 7.6 and is independent of agent concentration.

An alternative to ratiometric PARACEST is the use of linewidth analysis of a single CEST peak (Figure 17) [62, 63]. The linewidth of the CEST effect of Tm-DOTAM-Gly-Lys is independent of agent concentration for given saturation pulse conditions. Within the physiological range of $\mathrm{pH} 6.0-7.5$, linewidth analysis is less sensitive to temperature. The agent was directly injected into mouse's leg muscle and a $\mathrm{pH}$ value of $7.2 \pm 0.2$ was measured. This example also provides the possibility of conjugating DOTAM with peptides to improve pharmacokinetic properties such as high cellular uptake and longer intracellular retention $[62,63,104]$. However, this method of analysis is not suitable for overlapping CEST effects.
5.4. Exogenous DIACEST Agents. The first documented CEST MRI contrast agent was urea, which decreased the water proton signal in ex vivo kidney tissue [105]. As the chemical exchange of an amide proton with water is basecatalyzed, the CEST effect of urea was shown to be dependent on $\mathrm{pH}$ [106]. This CEST effect is characterized as diamagnetic CEST (DIACEST) as opposed to PARACEST agent with metal ions.

DIACEST agents typically have exchangeable protons with a chemical shift that is less than $10 \mathrm{ppm}$ from the water resonance. For example, a hydroxyl proton has a chemical shift of $\sim 1 \mathrm{ppm}$, an amine proton resonates at $\sim 2-3 \mathrm{ppm}$, and an amide proton typically has a MR frequency greater than $2.5 \mathrm{ppm}$, with the basic and aromatic proton shifted more down field [107]. The first reported DIACEST agent, 5,6dihydrouracil, has a ratio of CEST effects from the two amides that follows a sigmoidal relationship with $\mathrm{pH}$ [105]. 
TABLE 2: Summary of CEST methods to detect $\mathrm{pH}$.

\begin{tabular}{|c|c|c|c|c|c|c|c|}
\hline Agent & Range & Admin. & $\begin{array}{l}\text { Spatial res., } \\
\mathrm{cm}^{3}\end{array}$ & Temporal res. & $\begin{array}{c}\text { CEST } \\
\text { acquisition }\end{array}$ & Mouse model & Reference \\
\hline $\begin{array}{l}\text { Yb-DOTAM-Gly \& } \\
\text { Eu-DOTAM-Gly }\end{array}$ & $6.5-8.0$ & - & - & - & - & In vitro & {$[54]$} \\
\hline Pr-DOTAM-Gly & $5.5-7.5$ & - & - & - & - & In vitro & {$[55,56]$} \\
\hline Nd-DOTAM-Gly & $6-7.5$ & - & - & - & - & In vitro & {$[55]$} \\
\hline Eu-DOTAM-Gly & $6-7.5$ & - & - & - & - & In vitro & {$[55]$} \\
\hline $\begin{array}{l}\text { Dendrimer } \\
\text { Yb-DOTAM }\end{array}$ & $5-7$ & - & - & - & - & In vitro & {$[57]$} \\
\hline 5,6-Dihydrouracil & $6.5-7.0$ & - & - & - & - & In vitro & [58] \\
\hline $\mathrm{APT}$ & $\mathrm{pH}>5.0$ & Endogenous & $1.56 \times 10^{-3}$ & $\begin{array}{c}4+10 \mathrm{~s} \\
(2 \mathrm{offset})\end{array}$ & $\begin{array}{c}\text { CEST-EPI } \\
0.6 \mu \mathrm{T}\end{array}$ & $\begin{array}{c}\text { Ischemic rat brain, } \\
6.52 \pm 0.32\end{array}$ & [59] \\
\hline $\begin{array}{l}\text { APT with } \\
\text { creatine }\end{array}$ & $5.2-6.4$ & Endogenous & $2.5 \times 10^{-3}$ & $\begin{array}{l}7.5+15 s \\
(2 \text { offset })\end{array}$ & $\begin{array}{c}\text { CEST-EPI } \\
0.6 \mu \mathrm{T}\end{array}$ & Healthy human brain & {$[60]$} \\
\hline Yb-HPDO3A & $5.2-6.7$ & i.v. $1.2 \mathrm{mmol} / \mathrm{kg}$ & $2.2 \times 10^{-3}$ & $5 \mathrm{~min}$ & $\begin{array}{l}\text { CEST-RARE, } \\
24 \mu \mathrm{T}\end{array}$ & $\begin{array}{l}\text { Melanoma } \\
\text { Early } 2 \mathrm{~mm} \text { dia } 6.1 \\
\text { Late } 10 \mathrm{~mm} \text { dia } 5.8\end{array}$ & {$[61]$} \\
\hline $\begin{array}{l}\text { Tm-DOTAM-Gly- } \\
\text { Lys }\end{array}$ & $6.0-8.0$ & $\begin{array}{c}\text { Direct inj } 50 \mu \mathrm{L} \\
100 \mathrm{mM}\end{array}$ & $1.28 \times 10^{-3}$ & $26 \min 36 \mathrm{~s}$ & $\begin{array}{c}\text { CEST-RARE } \\
14 \mu \mathrm{T}\end{array}$ & $\begin{array}{l}\text { Leg muscle } \\
7.2 \pm 0.2\end{array}$ & {$[62,63]$} \\
\hline Yb-DO3A-oAA & $\begin{array}{c}6.1-7.75 \\
6.35-7.57 \\
6.34-7.82 \pm 0.21\end{array}$ & $\begin{array}{l}50 \mu \mathrm{L} \text { of } 60 \mathrm{mM} \\
\text { direct injection } \\
\text { into flank tumor } \\
\text { i.v. } 50 \mu \mathrm{L} \\
100 \mathrm{mM}\end{array}$ & $1.17 \times 10^{-3}$ & $5.2 \mathrm{~min}$ & $\begin{array}{l}\text { CEST-FISP } \\
20 \mu \mathrm{T}\end{array}$ & $\begin{array}{c}\text { MCF-7, } \\
\text { pHe }<6.5 \\
37^{\circ} \mathrm{C} \\
\text { MDA-MB-231 ROI: } \\
6.82 \pm 0.21 \\
\text { Pixel: } 6.8 \pm 0.4\end{array}$ & {$[64,65]$} \\
\hline Iopamidol & $5.5-7.7$ & $2.0 \mathrm{mM} / \mathrm{kg}$ & $4.39 \times 10^{-4}$ & $4 \min 18 \mathrm{~s}$ & $\begin{array}{c}\text { CEST-RARE } \\
3 \mu \mathrm{T}\end{array}$ & $\begin{array}{c}\text { Acute kidney injury of } \\
\text { mouse, healthy kidney } \\
6.71 \pm 0.16 \text {, after injury, } \\
\text { pH } 7.09 \pm 0.10\end{array}$ & {$[66,67]$} \\
\hline Iopromide & $6.2-7.2$ & $12 \mathrm{mM} / \mathrm{kg}$ & $1.25 \times 10^{-4}$ & $4 \mathrm{~min} 50 \mathrm{~s}$ & $\begin{array}{c}\text { CEST-FISP } \\
2.8 \mu \mathrm{T} \\
\end{array}$ & $\begin{array}{c}\text { MDA-MB-231 } \\
\text { pH 6.5-7.2 }\end{array}$ & {$[68]$} \\
\hline
\end{tabular}

Many $\mathrm{pH}$ dependent DIACEST agents have been investigated, including poly-L-lysine (single amide, $3.75 \mathrm{ppm}$ ) and polyamidoamine dendrimer (three amides, 3.4-3.6 ppm) [96], poly-L-arginine (guanidyl amide and amide, 1.8 and $3.6 \mathrm{ppm}$ resp.), and poly-L-threonine (hydroxyl and amide, 0.6 and 3.5 ppm, resp.) [58]. The DIACEST agents glycogen, $\mathrm{L}$-arginine, and poly-L-lysine have also been encapsulated in liposomes for lymph node mapping in vivo. No $\mathrm{pH}$ dependence on the CEST effect was reported for these liposomeencapsulated agents, but these pioneering studies offer new approaches for studying the spatial and temporal dynamics of complex biological systems. As another intriguing approach, the hydroxyl peak of poly-L-threonine has been shown to drop as a function of $\mathrm{pH}$, because the exchange rate becomes too fast compared to the chemical shift difference between water and the hydroxyl group at high $\mathrm{pH}$ [58].

Iopamidol (Isovue, Bracco Imaging S.p.A.) is a FDA approved contrast agent for CT/X-ray imaging that has been shown to be a DIACEST agent (Figure 18) [98, 108]. This agent has five hydroxyl protons, two amide protons that share the same MR frequency, and an additional amide proton with a different MR frequency. Due to the iodinated aryl ring, the amide protons have MR frequencies that are significantly shifted from the water frequency, which facilitates the detection of the CEST effects from these amide protons. The ratio of the CEST effects from these amide protons is correlated with $\mathrm{pH}$ in the range of 5.5-7.4 via a third-order polynomial function [66]. In vivo kidney $\mathrm{pH}$ mapping was performed with healthy mice that were acidified and alkalinized with ammonium chloride and bicarbonate and with mice that had acute kidney injury [67]. No $\mathrm{pH}$ mapping of a tumor was performed, presumably because the agent could not reach a sufficient concentration in the tumor for statistically significant CEST detection.

We have developed a similar CEST MRI method that uses a similar FDA approved CT/X-ray contrast agent, iopromide (Ultravist, Bayer Healthcare, Inc.) (Figure 19) [68]. The measurement of tumor acidosis with this method is known as "acidoCEST MRI." This approach uses a rapid FISP MRI acquisition protocol to compensate for the potentially rapid in vivo pharmacokinetics of the agent and processes the results using signal averaging, Gaussian filtering, cubic spline smoothing, and Lorentzian line shape fitting to detect both CEST effects from this agent. A $\log _{10}$ ratio of these CEST signals is linearly correlated with $\mathrm{pHe}$, without influence from the agent's concentration or other environmental effects such as the endogenous $T_{1}$ relaxation time of the tissue. This approach can be used to measure the average $\mathrm{pHe}$ 
of the tumor and can also be used to construct pixelwise maps of tumor pHe. To date, we have used acidoCEST MRI to measure the tumor pHe in MCF-7 and MDA-MB-231 breast cancer models; Raji, Ramos, and Granta 519 lymphoma models; and orthotopic and xenograft models of SKOV3 ovarian cancer, which demonstrates the broad applicability of this method. In addition, iopromide is clinically approved for CT diagnoses, saturation power, and durations needed for acidoCEST MRI have already been implemented in the clinic, and most clinical MRI instruments can perform FISP MRI studies. Therefore, acidoCEST MRI is poised for immediate translation to the clinic (Table 2).

\section{Conclusions}

The noninvasive measurement of $\mathrm{pH}$ has evolved from optical imaging, PET, EPR imaging, MR spectroscopy, and $T_{1}$-weighted MRI, towards the newer technique of CEST MRI. Improvements in CEST MRI pulse sequences and image analysis methods have provided the ability to perform relatively routine research studies. Methods using endogenous proteins to generate CEST, PARACEST agents, and DIACEST agents are each under continued development. The approaches that measure endogenous CEST signals or signals from DIACEST agents that are already clinically approved have great potential for clinical translation. These inevitable clinical studies may provide the foundation for providing improved diagnoses and rapid evaluations of early responses to anticancer therapies and treatments.

\section{Conflict of Interests}

The authors declare that there is no conflict of interests regarding the publication of this paper.

\section{Acknowledgments}

The authors would like to acknowledge the support from the Phoenix Friends of the Arizona Cancer Center, the Community Foundation of Southern Arizona, the Better than Ever Program, R01CA167183-01 and P50 CA95060. Liu Qi Chen was supported through the Anne Rita Monahan Foundation.

\section{References}

[1] D. Hanahan and R. A. Weinberg, "Hallmarks of cancer: the next generation," Cell, vol. 144, no. 5, pp. 646-674, 2011.

[2] A. J. Beddek, P. Rawson, L. Peng et al., "Profiling the metabolic proteome of bovine mammary tissue," Proteomics, vol. 8, no. 7, pp. 1502-1515, 2008.

[3] O. Warburg, "On the origin of cancer cells," Science, vol. 123, no. 3191, pp. 309-314, 1956.

[4] M. G. V. Heiden, L. C. Cantley, and C. B. Thompson, "Understanding the Warburg effect: the metabolic requirements of cell proliferation," Science, vol. 324, no. 5930, pp. 1029-1033, 2009.

[5] R. J. Gillies, N. Raghunand, M. L. Garcia-Martin, and R. A. Gatenby, "pH imaging," IEEE Engineering in Medicine and Biology Magazine, vol. 23, no. 5, pp. 57-64, 2004.
[6] R. J. Gillies, N. Raghunand, G. S. Karczmar, and Z. M. Bhujwalla, "MRI of the tumor microenvironment," Journal of Magnetic Resonance Imaging, vol. 16, no. 4, pp. 430-450, 2002.

[7] R. Martínez-Zaguilán, E. A. Seftor, R. E. B. Seftor, Y. W. Chu, R. J. Gillies, and M. J. C. Hendrix, "Acidic $\mathrm{pH}$ enhances the invasive behavior of human melanoma cells," Clinical and Experimental Metastasis, vol. 14, no. 2, pp. 176-186, 1996.

[8] R. J. Gillies and R. A. Gatenby, "Hypoxia and adaptive landscapes in the evolution of carcinogenesis," Cancer and Metastasis Reviews, vol. 26, no. 2, pp. 311-317, 2007.

[9] M. L. Marino, F. Lozupone, S. Fais, and A. de Milito, "Tumor acidity and malignancy: novel aspects in the design of antitumor therapy," Cancer, vol. 6, pp. 55-66, 2008.

[10] N. Raghunand, B. P. Mahoney, and R. J. Gillies, “Tumor acidity, ion trapping and chemotherapeutics: II. pH-dependent partition coefficients predict importance of ion trapping on pharmacokinetics of weakly basic chemotherapeutic agents," Biochemical Pharmacology, vol. 66, no. 7, pp. 1219-1229, 2003.

[11] N. Raghunand, R. Martínez-Zaguilán, S. H. Wright, and R. J. Gillies, "pH and drug resistance. II. Turnover of acidic vesicles and resistance to weakly basic chemotherapeutic drugs," Biochemical Pharmacology, vol. 57, no. 9, pp. 1047-1058, 1999.

[12] B. P. Mahoney, N. Raghunand, B. Baggett, and R. J. Gillies, "Tumor acidity, ion trapping and chemotherapeutics. I. Acid $\mathrm{pH}$ affects the distribution of chemotherapeutic agents in vitro," Biochemical Pharmacology, vol. 66, no. 7, pp. 1207-1218, 2003.

[13] N. Raghunand, X. He, R. van Sluis et al., "Enhancement of chemotherapy by manipulation of tumour $\mathrm{pH}$," British Journal of Cancer, vol. 80, no. 7, pp. 1005-1011, 1999.

[14] I. F. Robey, B. K. Baggett, N. D. Kirkpatrick et al., "Bicarbonate increases tumor $\mathrm{pH}$ and inhibits spontaneous metastases," Cancer Research, vol. 69, no. 6, pp. 2260-2268, 2009.

[15] A. S. Silva, J. A. Yunes, R. J. Gillies, and R. A. Gatenby, “The potential role of systemic buffers in reducing intratumoral extracellular $\mathrm{pH}$ and acid-mediated invasion," Cancer Research, vol. 69, no. 6, pp. 2677-2684, 2009.

[16] B. S. Ashby and M. B. Cantab, "pH studies in human malignant tumours," The Lancet, vol. 288, no. 7458, pp. 312-315, 1966.

[17] D. M. Prescott, H. C. Charles, J. M. Poulson et al., "The relationship between intracellular and extracellular $\mathrm{pH}$ in spontaneous canine tumors," Clinical Cancer Research, vol. 6, no. 6, pp. 25012505, 2000.

[18] J. L. Wike-Hooley, A. P. van den Berg, J. van der Zee, and H. S. Reinhold, "Human tumour $\mathrm{pH}$ and its variation," European Journal of Cancer and Clinical Oncology, vol. 21, no. 7, pp. 785791, 1985.

[19] R. A. Gatenby, E. T. Gawlinski, A. F. Gmitro, B. Kaylor, and R. J. Gillies, "Acid-mediated tumor invasion: a multidisciplinary study," Cancer Research, vol. 66, no. 10, pp. 5216-5223, 2006.

[20] Y. Lin, T.-Y. Wu, and A. F. Gmitro, "Error analysis of ratiometric imaging of extracellular $\mathrm{pH}$ in a window chamber model," Journal of Biomedical Optics, vol. 17, no. 4, Article ID 046004, 2012.

[21] V. Estrella, T. Chen, M. Lloyd et al., "Acidity generated by the tumor microenvironment drives local invasion," Cancer Research, vol. 73, no. 5, pp. 1524-1535, 2013.

[22] Y. K. Reshetnyak, M. Segala, O. A. Andreev, and D. M. Engelman, "A monomeric membrane peptide that lives in three worlds: in solution, attached to, and inserted across lipid bilayers," Biophysical Journal, vol. 93, no. 7, pp. 2363-2372, 2007. 
[23] A. L. Vāvere, G. B. Biddlecombe, W. M. Spees et al., "A novel technology for the imaging of acidic prostate tumors by positron emission tomography," Cancer Research, vol. 69, no. 10, pp. 4510-4516, 2009.

[24] D. Weerakkody, A. Moshnikova, M. S. Thakur et al., "Family of $\mathrm{pH}$ (low) insertion peptides for tumor targeting," Proceedings of the National Academy of Sciences of the United States of America, vol. 110, no. 15, pp. 5834-5839, 2013.

[25] C. Li, J. Xia, X. Wei, H. Yan, Z. Si, and S. Ju, "PH-Activated nearinfrared fluorescence nanoprobe imaging tumors by sensing the acidic microenvironment," Advanced Functional Materials, vol. 20, no. 14, pp. 2222-2230, 2010.

[26] M. Dellian, G. Helmlinger, F. Yuan, and R. K. Jain, "Fluorescence ratio imaging of interstitial $\mathrm{pH}$ in solid tumours: effect of glucose on spatial and temporal gradients," British Journal of Cancer, vol. 74, no. 8, pp. 1206-1215, 1996.

[27] M. Hassan, J. Riley, V. Chernomordik et al., "Fluorescence lifetime imaging system for in vivo studies," Molecular Imaging, vol. 6, no. 4, pp. 229-236, 2007.

[28] G. R. Martin and R. K. Jain, "Noninvasive measurement of interstitial $\mathrm{pH}$ profiles in normal and neoplastic tissue using fluorescence ratio imaging microscopy," Cancer Research, vol. 54, no. 21, pp. 5670-5674, 1994.

[29] R. A. Gatenby, E. T. Gawlinski, A. F. Gmitro, B. Kaylor, and R. J. Gillies, "Acid-mediated tumor invasion: a multidisciplinary study," Cancer Research, vol. 66, no. 10, pp. 5216-5223, 2006.

[30] W. Stummer, U. Pichlmeier, T. Meinel, O. D. Wiestler, F. Zanella, and H.-J. Reulen, "Fluorescence-guided surgery with 5-aminolevulinic acid for resection of malignant glioma: a randomised controlled multicentre phase III trial," The Lancet Oncology, vol. 7, no. 5, pp. 392-401, 2006.

[31] O. A. Andreev, A. D. Dupuy, M. Segala et al., "Mechanism and uses of a membrane peptide that targets tumors and other acidic tissues in vivo," Proceedings of the National Academy of Sciences of the United States of America, vol. 104, no. 19, pp. 7893-7898, 2007.

[32] E. P. Visser, J. A. Disselhorst, M. Brom et al., "Spatial resolution and sensitivity of the Inveon small-animal PET scanner," Journal of Nuclear Medicine, vol. 50, no. 1, pp. 139-147, 2009.

[33] T. Yoshitomi, R. Suzuki, T. Mamiya, H. Matsui, A. Hirayama, and Y. Nagasaki, "pH-sensitive radical-containing-nanoparticle (RNP) for the L-band-EPR imaging of low pH circumstances," Bioconjugate Chemistry, vol. 20, no. 9, pp. 1792-1798, 2009.

[34] M.-L. García-Martín, G. Hérigualt, C. Rémy et al., "Mapping extracellular $\mathrm{pH}$ in rat brain gliomas in vivo by ${ }^{1} \mathrm{H}$ magnetic resonance spectroscopic imaging: comparison with maps of metabolites," Cancer Research, vol. 61, no. 17, pp. 6524-6531, 2001.

[35] V. V. Khramtsov, I. A. Grigor'ev, M. A. Foster, D. J. Lurie, and I. Nicholson, "Biological applications of spin $\mathrm{pH}$ probes," Cellular and Molecular Biology, vol. 46, no. 8, pp. 1361-1374, 2000.

[36] A. Sotgiu, K. Mäder, G. Placidi, S. Colacicchi, C. L. Ursini, and M. Alecci, "pH-Sensitive imaging by low-frequency EPR: a model study for biological applications," Physics in Medicine and Biology, vol. 43, no. 7, pp. 1921-1930, 1998.

[37] B. Yoo and M. D. Pagel, "An overview of responsive MRI contrast agents for molecular imaging," Frontiers in Bioscience, vol. 13, no. 5, pp. 1733-1752, 2008.

[38] W. Golder, "Magnetic resonance spectroscopy in clinical oncology," Onkologie, vol. 27, no. 3, pp. 304-309, 2004.
[39] R. J. Gillies and D. L. Morse, "In vivo magnetic resonance spectroscopy in cancer," Annual Review of Biomedical Engineering, vol. 7, pp. 287-326, 2005.

[40] P. Vermathen, A. A. Capizzano, and A. A. Maudsley, "Administration and ${ }^{1} \mathrm{H}$ MRS detection of histidine in human brain: application to in vivo $\mathrm{pH}$ measurement," Magnetic Resonance in Medicine, vol. 43, no. 5, pp. 665-675, 2000.

[41] R. J. Gillies, Z. Liu, and Z. Bhujwalla, " ${ }^{31} \mathrm{P}-\mathrm{MRS}$ measurements of extracellular $\mathrm{pH}$ of tumors using 3-aminopropylphosphonate," The American Journal of Physiology, vol. 267, no. 1, pp. C195-C203, 1994.

[42] Y. Aoki, K. Akagi, Y. Tanaka, J. Kawai, and M. Takahashi, "Measurement of intratumor $\mathrm{pH}$ by $\mathrm{pH}$ indicator used in 19Fmagnetic resonance spectroscopy: measurement of extracellular $\mathrm{pH}$ decrease caused by hyperthermia combined with hydralazine," Investigative Radiology, vol. 31, no. 11, pp. 680-689, 1996.

[43] Z. M. Bhujwalla, C. L. McCoy, J. D. Glickson, R. J. Gillies, and M. Stubbs, "Estimations of intra- and extracellular volume and $\mathrm{pH}$ by 31P magnetic resonance spectroscopy: effect of therapy on RIF-1 tumours," British Journal of Cancer, vol. 78, no. 5, pp. 606-611, 1998.

[44] N. W. Lutz, Y. L. Fur, J. Chiche, J. Pouysse, and P. J. Cozzone, "Quantitative in vivo characterization of intracellular and extracellular $\mathrm{pH}$ profiles in heterogeneous tumors: a novel method enabling multiparametric $\mathrm{pH}$ analysis," Cancer Research, vol. 73, no. 15, pp. 4616-4628, 2013.

[45] R. Zhou, N. Bansal, D. B. Leeper, and J. D. Glickson, "Intracellular acidification of human melanoma xenografts by the respiratory inhibitor $\mathrm{m}$-iodobenzylguanidine plus hyperglycemia: a $31 \mathrm{P}$ magnetic resonance spectroscopy study," Cancer Research, vol. 60, no. 13, pp. 3532-3536, 2000.

[46] N. Raghunand, B. Mahoney, R. van Sluis, B. Baggett, and R. J. Gillies, "Acute metabolic alkalosis enhances response of $\mathrm{C}_{3} \mathrm{H}$ mouse mammary tumors to the weak base mitoxantrone," Neoplasia, vol. 3, no. 3, pp. 227-235, 2001.

[47] A. S. E. Ojugo, P. M. J. McSheehy, D. J. O. McIntyre et al., "Measurement of the extracellular $\mathrm{pH}$ of solid tumours in mice by magnetic resonance spectroscopy: a comparison of exogenous ${ }^{19} \mathrm{~F}$ and ${ }^{31} \mathrm{P}$ probes," NMR in Biomedicine, vol. 12, no. 8, pp. 495-504, 1999.

[48] F. A. Gallagher, M. I. Kettunen, S. E. Day et al., "Magnetic resonance imaging of $\mathrm{pH}$ in vivo using hyperpolarized ${ }^{13} \mathrm{C}$ labelled bicarbonate," Nature, vol. 453, no. 7197, pp. 940-943, 2008.

[49] M. Oishi, S. Sumitani, and Y. Nagasaki, "On-off regulation of ${ }^{19} \mathrm{~F}$ magnetic resonance signals based on $\mathrm{pH}$-sensitive PEGylated nanogels for potential tumor-specific smart ${ }^{19} \mathrm{~F}$ MRI probes," Bioconjugate Chemistry, vol. 18, no. 5, pp. 1379-1382, 2007.

[50] M. L. Garcia-Martin, G. V. Martinez, N. Raghunand, A. D. Sherry, S. Zhang, and R. J. Gillies, "High resolution pHe imaging of rat glioma using $\mathrm{pH}$-dependent relaxivity," Magnetic Resonance in Medicine, vol. 55, no. 2, pp. 309-315, 2006.

[51] N. Raghunand, C. Howison, A. D. Sherry, S. Zhang, and R. J. Gillies, "Renal and systemic $\mathrm{pH}$ imaging by contrast-enhanced MRI," Magnetic Resonance in Medicine, vol. 49, no. 2, pp. 249257, 2003.

[52] N. Raghunand, S. Zhang, A. D. Sherry, and R. J. Gillies, "In vivo magnetic resonance imaging of tissue $\mathrm{pH}$ using a novel $\mathrm{pH}$ sensitive contrast agent, GdDOTA-4AmP," Academic Radiology, vol. 9, no. 2, pp. S481-S483, 2002. 
[53] G. V. Martinez, X. Zhang, M. L. García-Martín et al., "Imaging the extracellular $\mathrm{pH}$ of tumors by MRI after injection of a single cocktail of $\mathrm{T}_{1}$ and $\mathrm{T}_{2}$ contrast agents," NMR in Biomedicine, vol. 24, no. 10, pp. 1380-1391, 2011.

[54] S. Aime, A. Barge, D. D. Castelli et al., "Paramagnetic lanthanide(III) complexes as $\mathrm{pH}$-sensitive chemical exchange saturation transfer (CEST) contrast agents for MRI applications," Magnetic Resonance in Medicine, vol. 47, no. 4, pp. 639-648, 2002.

[55] S. Aime, D. Delli Castelli, and E. Terreno, "Novel pH-reporter MRI contrast agents," Angewandte Chemie International Edition, vol. 41, no. 22, pp. 4334-4336, 2002.

[56] E. Terreno, D. D. Castelli, G. Cravotto, L. Milone, and S. Aime, "Ln(III)-DOTAMGly complexes: a versatile series to assess the determinants of the efficacy of paramagnetic chemical exchange saturation transfer agents for magnetic resonance imaging applications," Investigative Radiology, vol. 39, no. 4, pp. 235-243, 2004.

[57] J. A. Pikkemaat, R. T. Wegh, R. Lamerichs et al., "Dendritic PARACEST contrast agents for magnetic resonance imaging," Contrast Media and Molecular Imaging, vol. 2, no. 5, pp. 229239, 2007.

[58] M. T. McMahon, A. A. Gilad, M. A. DeLiso, S. M. Cromer Berman, J. W. M. Bulte, and P. C. M. van Zijl, "New 'multicolor' polypeptide diamagnetic chemical exchange saturation transfer (DIACEST) contrast agents for MRI," Magnetic Resonance in Medicine, vol. 60, no. 4, pp. 803-812, 2008.

[59] J. Zhou, J.-F. Payen, D. A. Wilson, R. J. Traystman, and P. C. M. Van Zijl, "Using the amide proton signals of intracellular proteins and peptides to detect $\mathrm{pH}$ effects in MRI," Nature Medicine, vol. 9, no. 8, pp. 1085-1090, 2003.

[60] P. Z. Sun, T. Benner, A. Kumar, and A. G. Sorensen, "Investigation of optimizing and translating $\mathrm{pH}$-sensitive pulsedchemical exchange saturation transfer (CEST) imaging to a 3T clinical scanner," Magnetic Resonance in Medicine, vol. 60, no. 4, pp. 834-841, 2008.

[61] D. Delli Castelli, G. Ferrauto, J. C. Cutrin, E. Terreno, and S. Aime, "In vivo maps of extracellular $\mathrm{pH}$ in murine melanoma by CEST-MRI," Magnetic Resonance in Medicine, vol. 71, no. 1, pp. 326-332, 2014.

[62] N. McVicar, A. X. Li, M. Suchý, R. H. E. Hudson, R. S. Menon, and R. Bartha, "Simultaneous in vivo $\mathrm{pH}$ and temperature mapping using a PARACEST-MRI contrast agent," Magnetic Resonance in Medicine, vol. 70, no. 4, pp. 1016-1025, 2013.

[63] F. Wojciechowski, M. Suchy, A. X. Li, H. A. Azab, R. Bartha, and R. H. E. Hudson, "A robust and convergent synthesis of dipeptide-DOTAM conjugates as chelators for lanthanide ions: new PARACEST MRI agents," Bioconjugate Chemistry, vol. 18, no. 5, pp. 1625-1636, 2007.

[64] P. Z. Sun, E. Wang, J. S. Cheung, X. Zhang, T. Benner, and A. G. Sorensen, "Simulation and optimization of pulsed radio frequency irradiation scheme for chemical exchange saturation transfer (CEST) MRI-demonstration of $\mathrm{pH}$-weighted pulsedamide proton CEST MRI in an animal model of acute cerebral ischemia," Magnetic Resonance in Medicine, vol. 66, no. 4, pp. 1042-1048, 2011.

[65] G. Liu, Y. Li, V. R. Sheth, and M. D. Pagel, "Imaging in vivo extracellular $\mathrm{pH}$ with a single paramagnetic chemical exchange saturation transfer magnetic resonance imaging contrast agent," Molecular Imaging, vol. 11, no. 1, pp. 47-57, 2012.

[66] D. L. Longo, W. Dastrù, G. Digilio et al., "Iopamidol as a responsive MRI-chemical exchange saturation transfer contrast agent for $\mathrm{pH}$ mapping of kidneys: in vivo studies in mice at $7 \mathrm{~T}$,' Magnetic Resonance in Medicine, vol. 65, no. 1, pp. 202-211, 2011.

[67] D. L. Longo, A. Busato, S. Lanzardo, F. Antico, and S. Aime, "Imaging the $\mathrm{pH}$ evolution of an acute kidney injury model by means of iopamidol, a MRI-CEST pH-responsive contrast agent," Magnetic Resonance in Medicine, vol. 70, no. 3, pp. 859864, 2013.

[68] L. Q. Chen, C. M. Howison, J. J. Jeffery, I. F. Robey, P. H. Kuo, and M. D. Pagel, "Evaluations of extracellular $\mathrm{pH}$ within in vivo tumors using acidoCEST MRI," Magnetic Resonance in Medicine, vol. 72, no. 5, pp. 1408-1417, 2014.

[69] P. A. Bottomley, "Spatial localization in NMR spectroscopy in vivo," Annals of the New York Academy of Sciences, vol. 508, pp. 333-348, 1987.

[70] J. Frahm, K.-D. Merboldt, and W. Hänicke, "Localized proton spectroscopy using stimulated echoes," Journal of Magnetic Resonance, vol. 72, no. 3, pp. 502-508, 1987.

[71] J. H. Ardenkjær-Larsen, B. Fridlund, A. Gram et al., "Increase in signal-to-noise ratio of $>10,000$ times in liquid-state NMR," Proceedings of the National Academy of Sciences of the United States of America, vol. 100, no. 18, pp. 10158-10163, 2003.

[72] S. Aime, M. Botta, S. G. Crich, G. Giovenzana, G. Palmisano, and M. Sisti, "A macromolecular Gd(III) complex as pHresponsive relaxometric probe for MRI applications," Chemical Communications, no. 16, pp. 1577-1578, 1999.

[73] R. Hovland, C. Gløgård, A. J. Aasen, and J. Klaveness, “Gadolinium DO3A derivatives mimicking phospholipids; preparation and in vitro evaluation as $\mathrm{pH}$ responsive MRI contrast agents," Journal of the Chemical Society, Perkin Transactions 2, no. 6, pp. 929-933, 2001.

[74] S. Laus, A. Sour, R. Ruloff, É. Tóth, and A. E. Merhach, "otational dynamics account for $\mathrm{pH}$-dependent relaxivities of PAMAM dendrimeric, Gd-based potential MRI contrast agents," Chemistry - A European Journal, vol. 11, no. 10, pp. 3064-3076, 2005.

[75] K. B. Hartman, S. Laus, R. D. Bolskar et al., "Gadonanotubes as ultrasensitive $\mathrm{pH}$-smart probes for magnetic resonance imaging," Nano Letters, vol. 8, no. 2, pp. 415-419, 2008.

[76] É. Tóth, R. D. Bolskar, A. Borel et al., "Water-soluble gadofullerenes: toward high-relaxivity, $\mathrm{pH}$-responsive MRI contrast agents," Journal of the American Chemical Society, vol. 127, no. 2, pp. 799-805, 2005.

[77] K.-E. Lokling, S. L. Fossheim, R. Skurtveit, A. Bjornerud, and J. Klaveness, "pH-sensitive paramagnetic liposomes as MRI contrast agents: in vitro feasibility studies," Magnetic Resonance Imaging, vol. 19, no. 5, pp. 731-738, 2001.

[78] M. P. Lowe, D. Parker, O. Reany et al., "pH-dependent modulation of relaxivity and luminescence in macrocyclic gadolinium and europium complexes based on reversible intramolecular sulfonamide ligation," Journal of the American Chemical Society, vol. 123, no. 31, pp. 7601-7609, 2001.

[79] M. Woods, G. E. Kiefer, S. Bott et al., "Synthesis, relaxometric and photophysical properties of a new $\mathrm{pH}$-responsive MRI contrast agent: the effect of other ligating groups on dissociation of a p-nitrophenolic pendant arm," Journal of the American Chemical Society, vol. 126, no. 30, pp. 9248-9256, 2004.

[80] J. Hall, R. Häner, S. Aime et al., "Relaxometric and luminescence behaviour of triaquahexaazamacrocyclic complexes, the gadolinium complex displaying a high relaxivity with a pronounced $\mathrm{pH}$ dependence," New Journal of Chemistry, vol. 22, no. 6, pp. 627-631, 1998. 
[81] S. Lecommandoux, O. Sandre, F. Chécot, and R. Perzynski, "Smart hybrid magnetic self-assembled micelles and hollow capsules," Progress in Solid State Chemistry, vol. 34, no. 2-4, pp. 171-179, 2006.

[82] F. M. Goycoolea, M. E. Fernández-Valle, I. Aranaz, and Á. Heras, "PH- and temperature-sensitive chitosan hydrogels: swelling and MRI studies," Macromolecular Chemistry and Physics, vol. 212, no. 9, pp. 887-895, 2011.

[83] K. T. Jokivarsi, H. I. Gröhn, O. H. Gröhn, and R. A. Kauppinen, "Proton transfer ratio, lactate, and intracellular $\mathrm{pH}$ in acute cerebral ischemia," Magnetic Resonance in Medicine, vol. 57, no. 4, pp. 647-653, 2007.

[84] P. Z. Sun, E. Wang, and J. S. Cheung, "Imaging acute ischemic tissue acidosis with $\mathrm{pH}$-sensitive endogenous amide proton transfer (APT) MRI-Correction of tissue relaxation and concomitant RF irradiation effects toward mapping quantitative cerebral tissue pH," NeuroImage, vol. 60, no. 1, pp. 1-6, 2012.

[85] S. Zhang, K. Wu, and A. D. Sherry, "A novel pH-sensitive MRI contrast agent," Angewandte Chemie-International Edition, vol. 38, no. 21, pp. 3192-3194, 1999.

[86] S. Aime, F. Fedeli, A. Sanino, and E. Terreno, "A R2/R1 ratiometric procedure for a concentration-independent, $\mathrm{pH}$ responsive, Gd(III)-based MRI agent," Journal of the American Chemical Society, vol. 128, no. 35, pp. 11326-11327, 2006.

[87] A. D. Sherry and M. Woods, "Chemical exchange saturation transfer contrast agents for magnetic resonance imaging," Annual Review of Biomedical Engineering, vol. 10, pp. 391-411, 2008.

[88] E. Vinogradov, A. D. Sherry, and R. E. Lenkinski, "CEST: from basic principles to applications, challenges and opportunities," Journal of Magnetic Resonance, vol. 229, pp. 155-172, 2013.

[89] P. C. M. van Zijl, C. K. Jones, J. Ren, C. R. Malloy, and A. D. Sherry, "MRI detection of glycogen in vivo by using chemical exchange saturation transfer imaging (glycoCEST)," Proceedings of the National Academy of Sciences of the United States of America, vol. 104, no. 11, pp. 4359-4364, 2007.

[90] W. Ling, R. R. Regatte, G. Navon, and A. Jerschow, "Assessment of glycosaminoglycan concentration in vivo by chemical exchange-dependent saturation transfer (gagCEST)," Proceedings of the National Academy of Sciences of the United States of America, vol. 105, no. 7, pp. 2266-2270, 2008.

[91] K. Cai, M. Haris, A. Singh et al., "Magnetic resonance imaging of glutamate," Nature Medicine, vol. 18, no. 2, pp. 302-306, 2012.

[92] J. Zhou, B. Lal, D. A. Wilson, J. Laterra, and P. C. M. van Zijl, "Amide proton transfer (APT) contrast for imaging of brain tumors," Magnetic Resonance in Medicine, vol. 50, no. 6, pp. 1120-1126, 2003.

[93] P. Z. Sun, J. Zhou, J. Huang, and P. Van Zijl, "Simplified quantitative description of amide proton transfer (APT) imaging during acute ischemia," Magnetic Resonance in Medicine, vol. 57, no. 2, pp. 405-410, 2007.

[94] P. Z. Sun, J. S. Cheung, E. Wang, and E. H. Lo, "Association between $\mathrm{pH}$-weighted endogenous amide proton chemical exchange saturation transfer MRI and tissue lactic acidosis during acute ischemic stroke," Journal of Cerebral Blood Flow and Metabolism, vol. 31, no. 8, pp. 1743-1750, 2011.

[95] J. Zhou, D. A. Wilson, P. Z. Sun, J. A. Klaus, and P. C. M. Van Zijl, "Quantitative description of proton exchange processes between water and endogenous and exogenous agents for WEX, CEST, and APT experiments," Magnetic Resonance in Medicine, vol. 51, no. 5, pp. 945-952, 2004.
[96] M. T. McMahon, A. A. Gilad, J. Zhou, P. Z. Sun, J. W. M. Bulte, and P. C. M. van Zijl, "Quantifying exchange rates in chemical exchange saturation transfer agents using the saturation time and saturation power dependencies of the magnetization transfer effect on the magnetic resonance imaging signal (QUEST and QUESP): $\mathrm{pH}$ calibration for poly-L-lysine and a starburst dendrimer," Magnetic Resonance in Medicine, vol. 55, no. 4, pp. 836-847, 2006.

[97] P. Z. Sun, Y. Murata, J. Lu, X. Wang, E. H. Lo, and A. G. Sorensen, "Relaxation-compensated fast multislice amide proton transfer (APT) imaging of acute ischemic stroke," Magnetic Resonance in Medicine, vol. 59, no. 5, pp. 1175-1182, 2008.

[98] M. Zaiss and P. Bachert, "Chemical exchange saturation transfer (CEST) and MR $Z$-spectroscopy in vivo: a review of theoretical approaches and methods," Physics in Medicine and Biology, vol. 58, no. 22, pp. R221-R269, 2013.

[99] S. Zhang, M. Merritt, D. E. Woessner, R. E. Lenkinski, and A. D. Sherry, "PARACEST agents: modulating MRI contrast via water proton exchange," Accounts of Chemical Research, vol. 36, no. 10, pp. 783-790, 2003.

[100] V. R. Sheth, G. Liu, Y. Li, and M. D. Pagel, "Improved pH measurements with a single PARACEST MRI contrast agent," Contrast Media and Molecular Imaging, vol. 7, no. 1, pp. 26-34, 2012.

[101] D. Dellicastelli, E. Terreno, and S. Aime, "Yb" ${ }^{I I I}-\mathrm{HPDO} A$ : a dual $\mathrm{pH}$ - and temperature-responsive CEST agent," Angewandte Chemie - International Edition, vol. 50, no. 8, pp. 1798 1800, 2011.

[102] V. R. Sheth, Y. Li, L. Q. Chen, C. M. Howison, C. A. Flask, and M. D. Pagel, "Measuring in vivo tumor $\mathrm{pHe}$ with CEST-FISP MRI," Magnetic Resonance in Medicine, vol. 67, no. 3, pp. 760768, 2012.

[103] Y. Wu, T. C. Soesbe, G. E. Kiefer, P. Zhao, and A. D. Sherry, "A responsive europium(III) chelate that provides a direct readout of $\mathrm{pH}$ by MRI," Journal of the American Chemical Society, vol. 132, no. 40, pp. 14002-14003, 2010.

[104] M. Suchý, A. X. Li, M. Milne, R. Bartha, and R. H. E. Hudson, "DOTAM-type ligands possessing arginine pendant groups for use in PARACEST MRI," Contrast Media and Molecular Imaging, vol. 7, no. 5, pp. 441-449, 2012.

[105] K. M. Ward and R. S. Balaban, "Determination of $\mathrm{pH}$ using water protons and chemical exchange dependent saturation transfer (CEST)," Magnetic Resonance in Medicine, vol. 44, no. 5, pp. 799-802, 2000.

[106] S. D. Wolff and R. S. Balaban, "Magnetization transfer contrast (MTC) and tissue water proton relaxation in vivo," Magnetic Resonance in Medicine, vol. 10, no. 1, pp. 135-144, 1989.

[107] K. M. Ward, A. H. Aletras, and R. S. Balaban, "A new class of contrast agents for MRI based on proton chemical exchange dependent saturation transfer (CEST)," Journal of Magnetic Resonance, vol. 143, no. 1, pp. 79-87, 2000.

[108] S. Aime, L. Calabi, L. Biondi et al., "Iopamidol: exploring the potential use of a well-established x-ray contrast agent for MRI," Magnetic Resonance in Medicine, vol. 53, no. 4, pp. 830-834, 2005. 


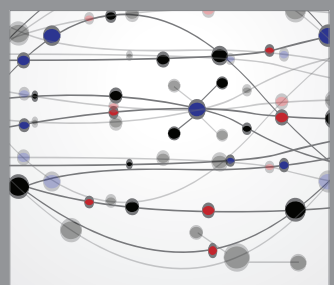

The Scientific World Journal
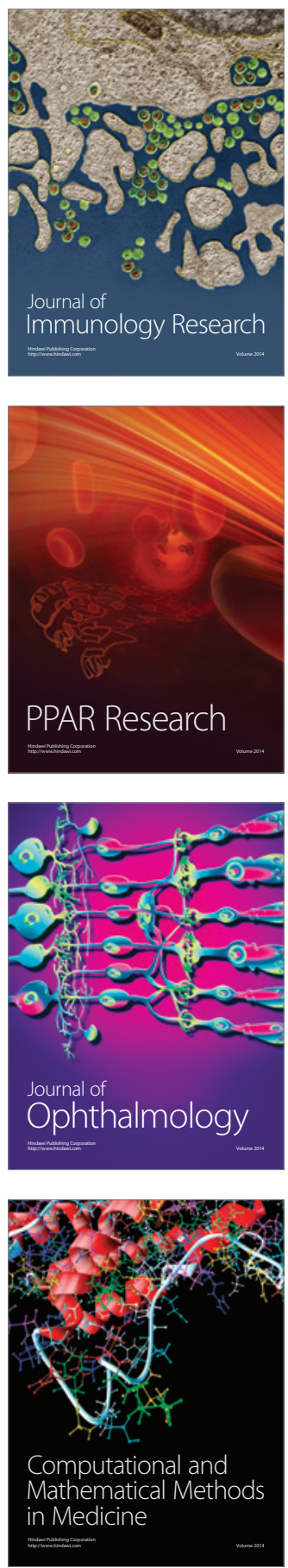

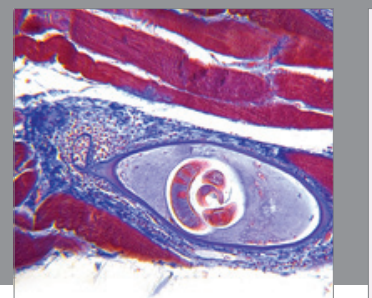

Gastroenterology

Research and Practice
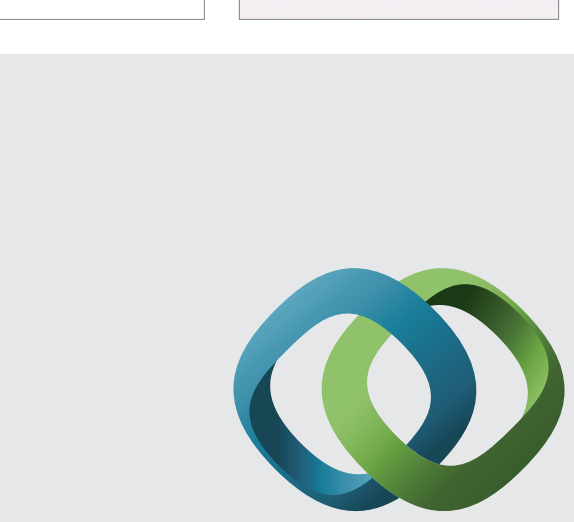

\section{Hindawi}

Submit your manuscripts at

http://www.hindawi.com
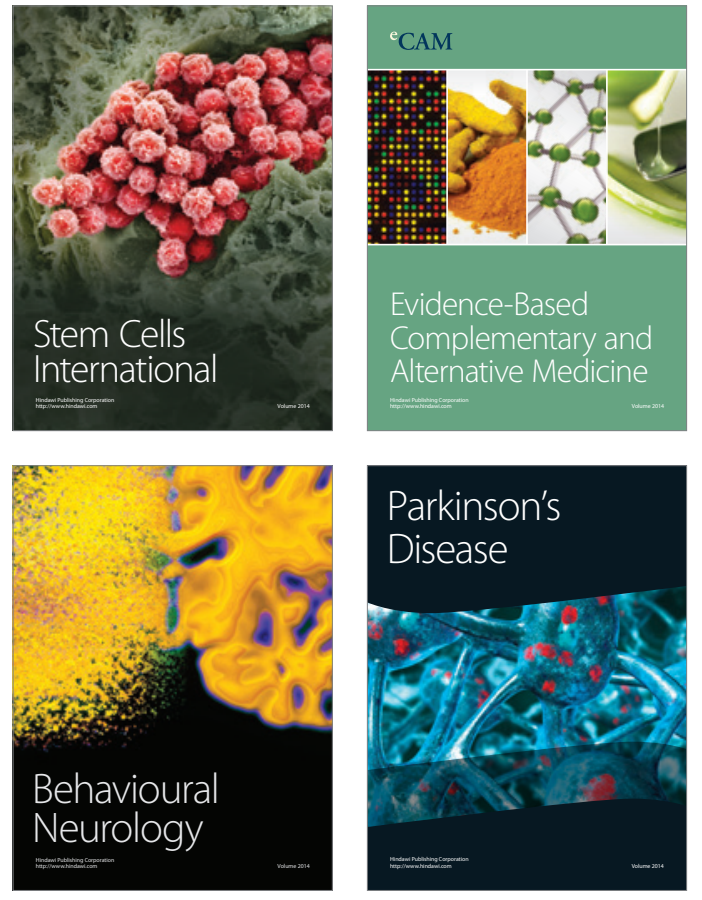
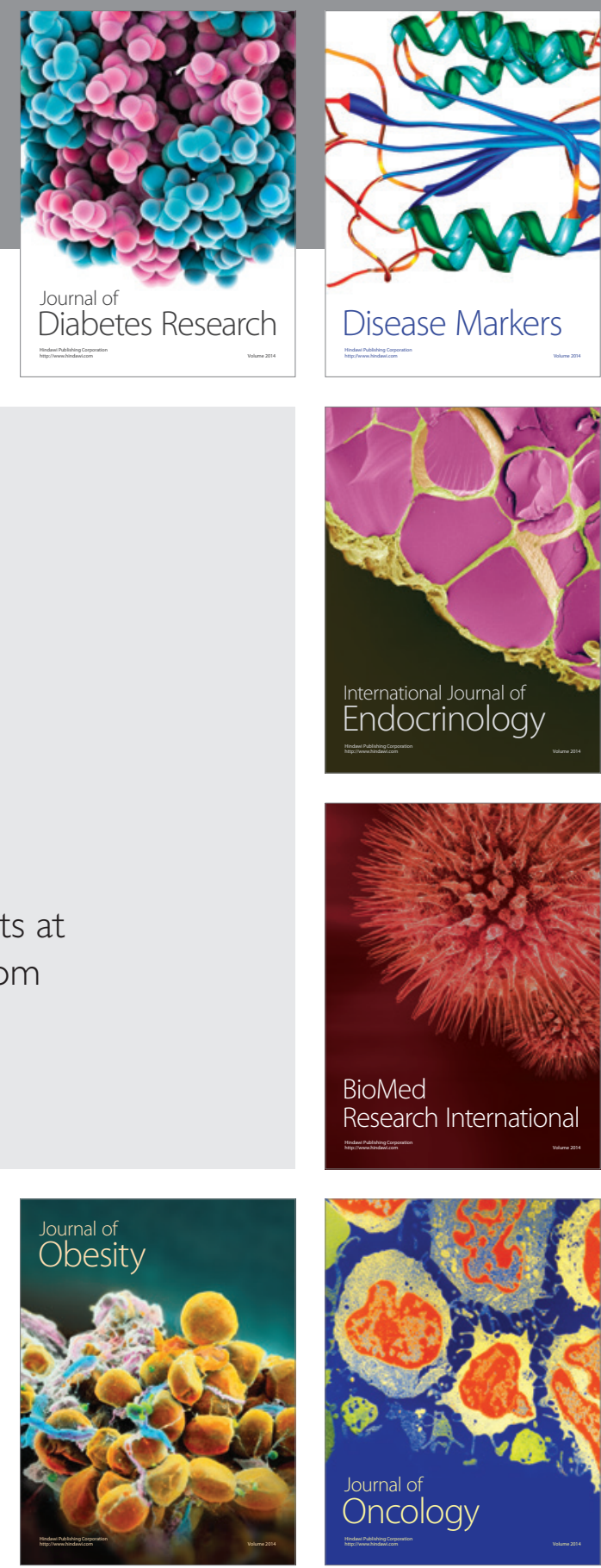

Disease Markers
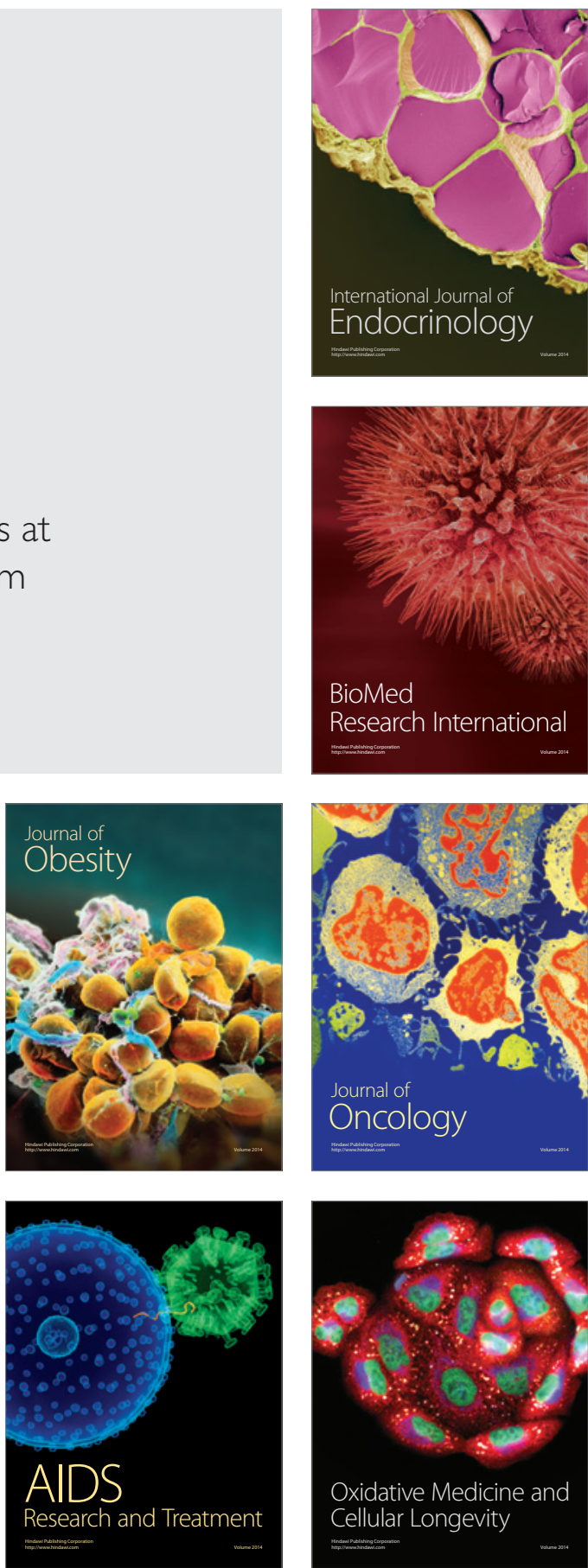